Title: Ocean model response to stochastically perturbed momentum fluxes

Authors:

Terence J. O'Kane

CSIRO Oceans and Atmosphere, Hobart, Tasmania, Australia terence.okane@csiro.au

Russell Fiedler

CSIRO Oceans and Atmosphere, Hobart, Tasmania, Australia russell.fiedler@,csiro.au

Mark A. Collier

CSIRO Oceans and Atmosphere, Aspendale, Victoria, Australia mark.collier@csiro.au

Vassili Kitsios

CSIRO Oceans and Atmosphere, Aspendale, Victoria, Australia

Laboratory for Turbulence Research in Aerospace and Combustion, Department of Mechanical and Aerospace Engineering Monash University, Clayton, Victoria 3800, Australia

vassili.kitsios@csiro.au

This is a non-peer reviewed preprint submitted to EarthArXiv.

The preprint has been submitted to the AMS Journal of Climate for peer review. 


\section{Ocean model response to stochastically perturbed momentum fluxes}

Terence J. O’Kane, ${ }^{\text {a }}$ Russell Fiedler, ${ }^{a}$ Mark A. Collier, ${ }^{b}$ Vassili Kitsios, ${ }^{\text {b,c }}$

${ }^{\text {a } C S I R O}$ Oceans and Atmosphere, Hobart, Tasmania, Australia

${ }^{\mathrm{b}}$ CSIRO Oceans and Atmosphere, Aspendale, Victoria, Australia

${ }^{\mathrm{c}}$ Laboratory for Turbulence Research in Aerospace and Combustion, Department of Mechanical and Aerospace Engineering Monash University, Clayton, Victoria 3800, Australia

7 Corresponding author: Terence J. O’Kane, terence.okane@csiro.au 
$\&$ ABSTRACT: In climate model configurations, standard approaches to the representation of unresolved, or subgrid scales, via deterministic closure schemes are being challenged by stochastic approaches inspired by statistical dynamical theory. Despite gaining popularity, studies of various stochastic subgrid scale parameterizations applied to atmospheric climate and weather prediction systems have revealed a diversity of model responses, including degeneracy in the response to different forcings and compensating model errors, with little reduction in artificial damping of the small scales required for numerical stability. Due to the greater range of spatio-temporal scales involved, how to best sample subgrid fluctuations in a computationally inexpensive manner, with the aim of reduced model error and improvements to the simulated climatological state of the ocean, remains an open question. While previous studies have considered perturbations to the surface forcing or subsurface temperature tendencies, we implement an energetically consistent, simple, stochastic subgrid eddy parameterization of the momentum fluxes in regions of the threedimensional ocean typically associated with high eddy variability. We consider the changes in the modelled energetics of low-resolution simulations in response to stochastically forced velocity tendencies whose perturbation statistics and amplitudes are calculated from an eddy resolving ocean configuration. Kinetic energy spectra from a triple-decomposition reveal a systematic redistribution from the seasonal (climatological minus mean) potential energy to preferentially generate small scale transient kinetic energy while the total energy spectra remains largely unchanged. We show that stochastic parameterization generally improves model biases, noticeably so for the simulated energetics of the Southern Oceans. 


\section{Introduction}

The question of how to incorporate the effects of unresolved turbulent motions, and their role in determining large scale dynamics, represents a common problem in large eddy simulations (LES) of nonlinear fluids and one that is particularly crucial for simulating geophysical flows. In ocean and climate modelling it is typical to employ deterministic methods which, due to the computational cost, often requires reduced resolution model configurations to enable long time simulations whereby only the statistical effects of the subgrid scales (eddies) on the retained large scales (mean flow) can be approximated empirically. Furthermore, it is well known that small scale errors grow rapidly on (finite) timescales determined by their initial spatial structure, where even small random errors will quickly become organised by the model dynamics and undergoe rapid growth and projection onto large scale mean features of the flow. The structure and growth rate of small scale errors is not confined to subgrid parameterizations but to all aspects of simulating and predicting geophysical flows (Kalnay 2003).

It has long been recognised that elements of the climate system might be represented by reduced order (linear) stochastic models, of which the principal oscillation or linear inverse model (Hasslemann 1976) is a classical example with a long history of application to ocean dynamics (Frankignoul and Hasslemann 1977; Penland 1989; Penland and Sardeshmukh 1995; Lou et al. 2021). More recently data driven approaches have been developed and applied for the construction of nonstationary reduced order stochastic models (Metzner et al. 2012) of the atmosphere (Horenko 2010) allowing for the identification of persistent regime behaviour such as that associated with the low frequency variability of the North Atlantic Oscillation (Quinn et al. 2021). These approaches to modeling geophysical flows assume scale separation i.e., that only a subset of large scale modes need to be resolved and that the subgrid scales may be represented in terms of stochastic noise forcing.

A foundational understanding of subgrid parameterizations to correct biases in the small scale energy spectra of LES has deep roots in statistical dynamics. As discussed by O'Kane and Frederiksen (2008a), fundamental insights into stochastic-dynamic parameterization were pionereed by the efforts of a key group working on turbulent energy closures for ensemble weather prediction. Specifically, the work of Epstein (1969), Fleming (1971a,b) and Pitcher (1977) (see also Epstein and Pitcher (1972)) in which third and higher order cumulants are discarded in order to 
directly forecast mean and variance information via statistical dynamical prognostic equations and stochastic perturbations to velocity tendencies. However, it was the seminal work of Kraichnan (1976) that marked the arrival of the modern theory of eddy viscosity and stochastic backscatter i.e. injection and or drain of energy with a predetermined renormalised functional form. Since then, there have been ongoing efforts over several decades to establish a rigorous mathematical basis for subgrid scale parameterizations based on statistical mechanics and dynamics, including formal renormalization methods (Frederiksen 1999; O'Kane and Frederiksen 2008b), stochastic approximations (Zidihkeri and Frederiksen 2008) and the subsequent identification of universal scaling laws for subgrid dynamics in atmospheric and oceanic flows (Kitsios et al. 2016). For a comprehensive review of the development of statistical dynamics and closures see the recent review by Zhou (2021). Various approaches to incorporating stochastic kinetic energy backscatter have for some time now been applied to reduce systematic model errors in operational weather prediction and atmospheric climate models (Berner et al. 2012; Franzke et al. 2015; Berner and coauthors 2017).

The aforementioned approaches seek to identify the scale dependent functional form of drain and injection terms in order to correct the energy spectrum of the smallest resolved scales in LES. One unavoidable consequence of the addition of stochastic forcing to a nonlinear system is that, typically, the amplitude of the noise increases with wavenumber hence making the small scales more isotropic and weakening phase relationships. In this case, whereas the energy spectrum may be improved, structure is lost. Additionally, it is often unclear the spatio-temporal scales at which the model will organise the noise and hence there is no a priori way to determine the coherent response to the forcing. Simply put, it remains unclear as to how any given nonlinear dynamical system will respond to a particular application of stochastic forcing.

Stochastic forcing can act in many ways to modify the dynamics of a nonlinear system. Examples include regime transitions in simple scalar systems such as the stochastically forced double well potential (Miller et al. 1985). In two-dimensional turbulence, weak stochastic forcing of a particular large scale mode or particular small wavenumber has been shown to be able to initiate large energy transfers from small to large scales via the inverse energy cascade (Bouchet and Simonnet 2009; Nadiga and O'Kane 2017). More generally, it has for some time now been recognised that stochastic forcing of the ocean surface fluxes, even isotropic random perturbations with zero mean, 
interacting with nonlinearities in the (climate) model equations can lead to enhanced variability and changes in the mean (climatological) state (Zavala-Garay et al. 2003; Beena and von Storch 2009; Williams 2012). Williams et al. (2016) showed the response to zero mean multivariate stochastic perturbations to the temperature tendencies in the three dimensional ocean. They considered both isotropic uncorrelated and correlated noise forcing whose amplitude was calculated from a $1 / 3^{\circ}$ horizontal resolution 40 vertical level climate ocean model to stochastically force the ocean temperature tendencies of a very low resolution $2.5^{\circ}$ latitude and $3.75^{\circ}$ longitude, 20 vertical level model. They found a stronger response occurred for correlated noise and with significant warming of the upper ocean and cooling at depth such that an overall significant loss of global ocean heat content occurred. Overall, they argue that perturbed temperature tendencies resulted in reduced biases and improved ocean temperature and salinity fields both at the surface and at depth, as well as improvements in the variability of the strength of the global ocean thermohaline circulation. The choice to perturb temperature tendencies in the three dimensional ocean state is consistent with well established reduced order models for examining ocean predictability (Lou et al. 2021) and applications examining initialization for ensemble ocean forecasting based on optimal perturbations to temperature (O'Kane et al. 2011). However, perturbing temperature alone is potentially problematic for large density compensated regions of the ocean and is inconsistent with energetics i.e. potential energy transfers of the form $u^{\prime} \rho^{\prime} \frac{\partial \rho}{\partial x}$.

O'Kane et al. (2013) showed that forcing of a low resolution Southern Ocean via the observed synoptic scale anomalous surface winds alone could account for the majority of the simulated variability in the subsurface Antarctic Circumpolar Current. Subsequently, O'Kane et al. (2014b) showed that even very weak stochastic forcing of the ocean surface winds can lead to excitation of chaotic oscillations in temperature and salinity in a low resolution ocean model, particularly in regions typically associated with tropical instability waves and subtropical baroclinic Rossby waves (O'Kane et al. 2014a; Chapman et al. 2020). However, there remains much uncertainty as to how best to apply, or even whether to apply, stochastic forcing to the three-dimensional state of a particular ocean or climate general circulation model (GCM) and what the modelled response might be.

Here we apply stochastic perturbations to the horizontal momentum flux in a general circulation ocean - sea ice model configuration with resolution typical for climate simulations. We argue that 
direct stochastic forcing of the velocity tendencies is more consistent with statistical dynamical theory and more aligned with applications in weather prediction. Specifically, a high resolution $1 / 10^{\circ}$ eddy resolving reference calculation forced by nominal year surface boundary conditions is used to determine regions of high eddy variance that are unresolved in the low resolution model and to set the amplitude of the applied stochastic perturbations. A low resolution $1^{\circ}$ control simulation is first run to steady state, also forced by nominal year surface boundary conditions, after which a series of simulations with stochastic perturbations to the horizontal momentum flux are conducted. The perturbation amplitudes are applied as a fraction of the variance of the reference eddy variability. A control simulation is run out to steady state $(\approx 2000$ years), then each of the stochastically perturbed simulations are also run to steady state, which is achieved after $\approx 150$ years, and continued for another two decades. The final decade of each of the 170 year simulations was used to examine the climatological (mean) ocean states, energetics and transports.

We describe the model configurations and construction of the stochastic forcing in section 2 . Results for a range of diagnostics are presented in section 3 followed by summary and discussion in section 4 .

\section{Experimental design and model configuration}

\section{a. Model configurations}

We employ the ACCESS-OM community model (Kiss et al. 2020) driven by JRA55-do repeat year forcing (Stewart et al. 2020) at two horizontal resolutions i.e. nominally $1^{\circ}$ and $0.1^{\circ}$. These models have been configured with model parameters as consistent as possible to assist in studies of resolution dependence. Away from the continental shelf and equatorward of $50^{\circ}$, the $0.1^{\circ}$ model resolves the first baroclinic deformation radius indicating some degree of representation of a transient mesoscale eddy field, whereas the $1^{\circ}$ does not. The low and high resolution models have different vertical resolutions where the vertical grid in the ACCESS-OM2 $1^{\circ}$ configuration has 50 levels and $2.3 \mathrm{~m}$ spacing at the surface, increasing smoothly to $219.6 \mathrm{~m}$ by the bottom at $5363.5 \mathrm{~m}$, whereas the ACCESS-OM2 $0.1^{\circ}$ configuration has 75 levels and $1.1 \mathrm{~m}$ spacing at the surface, increasing smoothly to $198.4 \mathrm{~m}$ by the bottom at $5808.7 \mathrm{~m}$. Kiss et al. (2020) provide a detailed description of the model parameters and performance of ACCESS-OM2 at three horizontal resolutions i.e. $1^{\circ}, 0.25^{\circ}$ and $0.1^{\circ}$. 


\section{b. Stochastic forcing}

Firstly, annual and seasonally varying climatological root mean squared errors (rmse) are calculated from the horizontal velocities using the final 10 years of a long control simulation of the ACCESS-OM $0.1^{\circ}$ model. The rmse are limited to only those values exceeding $0.15 \mathrm{~ms}^{-1}$ then regridded to the ACCESS-OM $1^{\circ}$ grid. The instantaneous zonal and meridional velocity tendencies $\left(\frac{\partial u}{\partial t}, \frac{\partial v}{\partial t}\right)$ are then perturbed by the addition of a random fluctuation $\left(\epsilon_{u}, \epsilon_{v}\right)$ uniformly distributed between $[-1,1]$ with zero mean and scaled to be some fraction of the rmse of the ACCESS-OM $0.1^{\circ}$ model. In this way, the tensorial flux form of the momentum equations in a curvilinear Z-coordinate system (Madec and the NEMO team 2016) are now given by

$$
\begin{array}{r}
\frac{\partial u}{\partial t}=\left(f+\frac{1}{e_{1} e_{2}}\left(v \frac{\partial e_{2}}{\partial i}-u \frac{\partial e_{1}}{\partial j}\right)\right) v \\
-\frac{1}{e_{1} e_{2}}\left(\frac{\partial\left(e_{2} u^{2}\right)}{\partial i}+\frac{\partial\left(e_{1} v u\right)}{\partial j}\right)-\frac{1}{e_{3}} \frac{\partial(w u)}{\partial k} \\
-\frac{1}{e_{1}} \frac{\partial}{\partial i}\left(\frac{p_{s}+p_{h}}{\rho_{o}}\right)+\epsilon_{u}+(\text { subgrid terms }+ \text { surface forcing }) \\
\frac{\partial v}{\partial t}=-\left(f+\frac{1}{e_{1} e_{2}}\left(v \frac{\partial e_{2}}{\partial i}-u \frac{\partial e_{1}}{\partial j}\right)\right) u \\
-\frac{1}{e_{1} e_{2}}\left(\frac{\partial\left(e_{2} u v\right)}{\partial i}+\frac{\partial\left(e_{1} v^{2}\right)}{\partial j}\right)-\frac{1}{e_{3}} \frac{\partial(w v)}{\partial k} \\
-\frac{1}{e_{2}} \frac{\partial}{\partial j}\left(\frac{p_{s}+p_{h}}{\rho_{o}}\right)+\epsilon_{v}+(\text { subgrid terms }+ \text { surface forcing })
\end{array}
$$

where $(i, j, k)$ are orthogonal curvilinear coordinates on the sphere associated with the positively oriented orthogonal set of unit vectors $(\mathbf{i}, \mathbf{j}, \mathbf{k})$ such that $\mathbf{k}$ is the local upward vector and $(\mathbf{i}, \mathbf{j})$ are two vectors orthogonal to $\mathbf{k}$ along geopotential surfaces. Here $(\lambda, \varphi, z)$ define the geographical coordinate system where position is defined by the latitude $\varphi(i, j)$, the longitude $\lambda(i, j)$ and the distance from the centre of the earth $a+z(k)$ and where $a$ is the earth's radius and $z$ the altitude above a reference sea level. The local deformation of the curvilinear coordinate system is then 
TABLE 1. Model configuration and amplitude of stochastic forcing as a percentage of the standard deviation from the ACCESS-OM2-0.1 high resolution reference simulation.

\begin{tabular}{ccc}
\hline \hline Model & resolution & amplitude \\
\hline ACCESS-OM2-0.1 & $1 / 10^{\circ}$ & 0 \\
ACCESS-OM2-1 (control) & $1^{\circ}$ & 0 \\
stochastic-1 & $1^{\circ}$ & $10 \%$ \\
stochastic-2 & $1^{\circ}$ & $20 \%$ \\
stochastic-5 & $1^{\circ}$ & $50 \%$ \\
stochastic-10 & $1^{\circ}$ & $100 \%$ \\
\hline
\end{tabular}

given by $e_{1}, e_{2}$ and $e_{3}$, three scale factors defined as

$$
\begin{array}{r}
e_{1}=(a+z)\left[\left(\frac{\partial \lambda}{\partial i} \cos \phi\right)^{2}+\left(\frac{\partial \phi}{\partial i}\right)^{2}\right]^{1 / 2} \\
e_{2}=(a+z)\left[\left(\frac{\partial \lambda}{\partial j} \cos \phi\right)^{2}+\left(\frac{\partial \phi}{\partial j}\right)^{2}\right]^{1 / 2} \\
e_{3}=\left(\frac{\partial z}{\partial k}\right)
\end{array}
$$

The masks are three dimensional with surface values of the zonal and merdional injection velocity amplitudes shown in figures 1a \& b. Specifically, we show the amplitude (mean) for the meridional and zonal velocity tendency forcing at the surface and also for the zonal velocities down to $300 \mathrm{~m}$ depth along the equator (figure 1c). The inset in figure 1a) illustrates the stochastic forcing on the meridional velocity tendency at a particular instant after regridding. The stochastic forcing is applied at each model timestep.

In the experiments that follow we consider stochastic forcing strengths of $10 \%, 20 \%, 50 \%$ and $100 \%$ of the regridded $0.1^{\circ}$ amplitude RMSE of anomalies with respect to climatology on the tendencies, and compared to a control simulations of the $1^{\circ}$ model and a reference $0.1^{\circ}$ model simulation as described in table 1. We further note that the decorrelation timescales for the velocities are significantly shorter than for temperature which is an important difference between this experimental design and that of earlier works where only temperature tendencies were perturbed (Williams et al. 2016) or where SST perturbations have been directly applied to analysed states (Andrejczuk et al. 2016). 

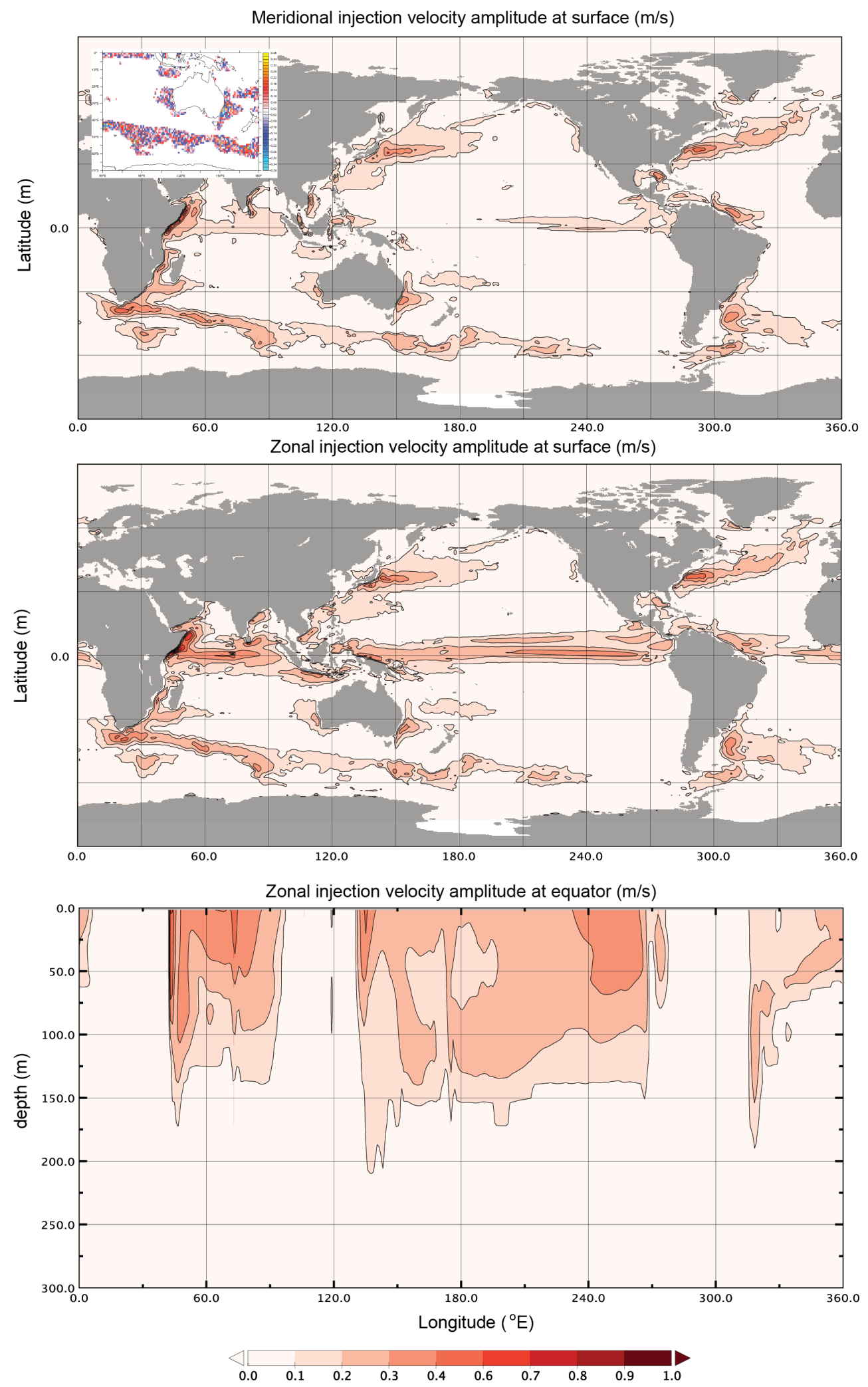

FIG. 1. Masks for stochastic perturbations to the velocity tendencies. The inset into the top panel shows the spatial distribution of instantaneous random values distributed between $[-1,1]$ within the mask about Australia and the Southern Ocean. 


\section{Results}

\section{a. Velocities}

In figures 2 we show the surface zonal and meridional velocity averaged over each January of the last decade of the respective 170 year simulations with JRA55-do repeat year forcing applied. All $1^{\circ}$ model simulations have started from the same initial conditions and all anomalies are relative to each respective model climatology calculated over a period where models are very close to steady state. In addition to an unperturbed control simulation (upper middle panels), we also include a $0.1^{\circ}$ reference simulation ACCESS-OM2-0.1 (upper left panel). The remaining panels in figures 2 show differences between the respective stochastically perturbed and control $1^{\circ}$ simulations.

Of immediate note for the zonal velocities, is the good correspondence between the broad features of the high resolution reference and low resolution control simulations. This is expected, given both models are driven with the same surface forcing. Also to be expected, is the absence of high amplitude, small scale features in the low resolution control simulation, and in particular in the Antarctic Circumpolar Current (ACC) and in the midlatitude boundary current regions such as the Kuroshio and Gulf Stream. In comparison to the ACCESS-OM2-1 control, it is the tropics, and in particular the Indonesian Through Flow (ITF) and Indian Ocean that respond most immediately to the applied stochastic forcing. The responses seen in the $10 \%$ stochastic- 1 and $20 \%$ stochastic-2 simulations are in the tropical instability waves in the equatorial Pacific and Atlantic, an equatorward displacement of the current associated with the ITF and similarly with the Indian ocean storm track extending from the Western Australian coast (O'Kane et al. 2014a; Chapman et al. 2020). As the amplitude of the perturbations is increased, we continue to see a strong response in the tropics but also responses in the Kuroshio and Gulf Stream associated with a poleward displacement of their separation and extensions.

For the meridional velocity there is a similar close correspondence between the broad scale structures of the respective high resolution reference and low resolution control simulations. There is a strong response in the equatorial Pacific at $240^{\circ}$ longitude, evident for even very weak stochastic forcing. This region has been previously identified by O'Kane et al. (2014b) to be characterised by high intrinsic variability and a strong sensitivity to stochastic atmospheric forcing. As the strength of the perturbation amplitude is increased there emerges responses at the midlatitudes 

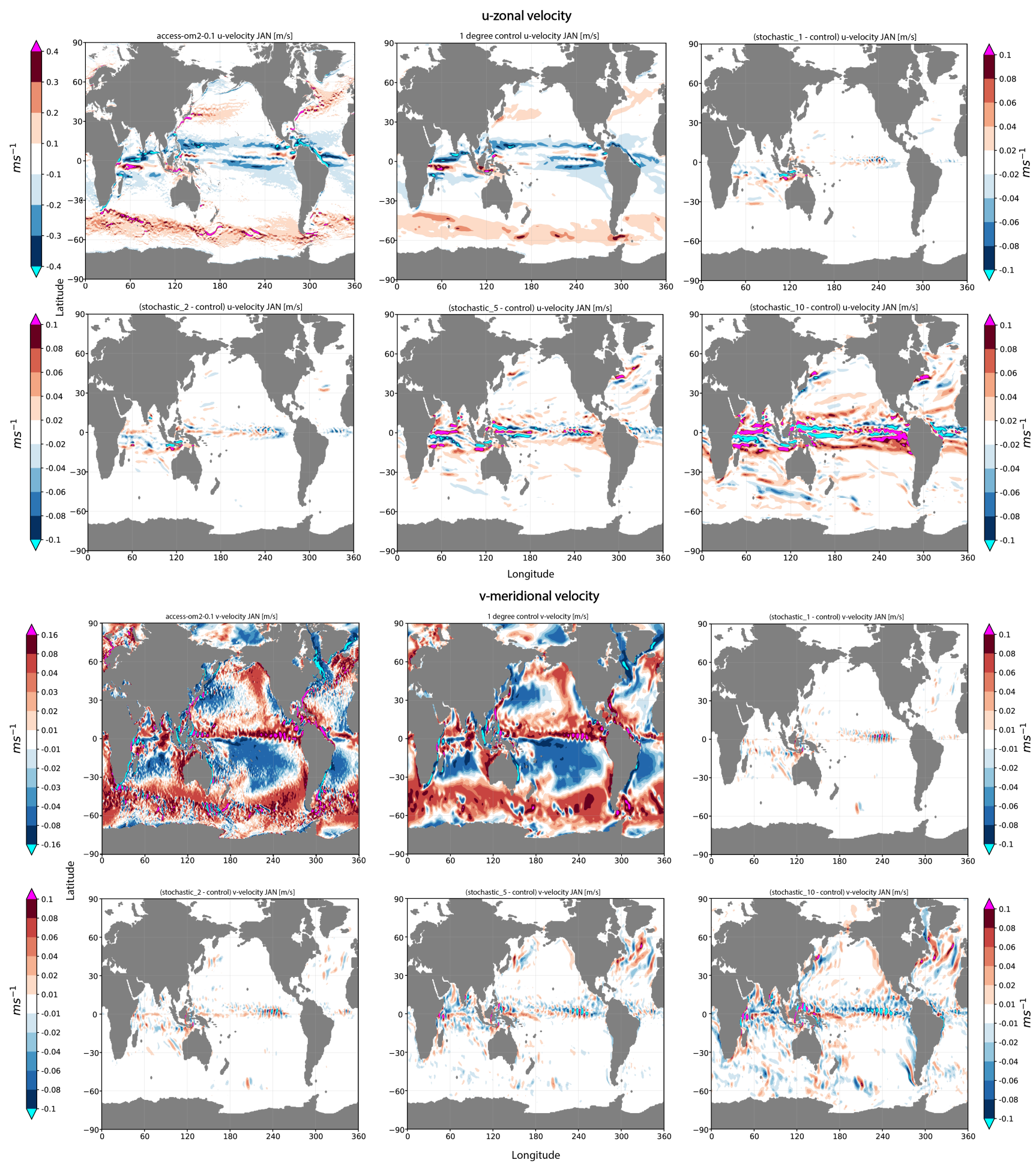

FIG. 2. High and low resolution zonal and meridional surface velocities averaged over last decade of 170 year 185 simulation and differences between stochastic and control simulations. 
and in particular located wherever major topographic features are present. For example, in the Southern Ocean in the ACC we see significant shifts in the meridional velocities in the vacinity of the East Pacific Rise. This region has previously been noted as one where intrinsic variability can be excited by reanalysed synoptics scale atmospheric surface ( 10 meter) winds alone (O'Kane et al. 2013). In the northern hemisphere, for the higher amplitude perturbations, there are significant responses across the entire North Atlantic and a westward shift in the Kuroshio separation.

\section{b. Energy}

The primary reason to perturb the velocity tendencies is to modify the momentum flux and the energetics. The total energy tendency (Orlanski and Cox 1973; Oey 2007) can be written as

$$
\frac{d}{d t}(E K E+E P E)=-\nabla \cdot\left(\overline{\mathbf{v}^{\prime} p^{\prime} / \rho_{0}}\right)+B T+B C+K H
$$

Here

$$
\begin{array}{r}
E K E=\frac{1}{2}\left(\overline{u^{\prime 2}}+\overline{v^{\prime 2}}\right) \\
E P E=\frac{g^{2}}{2 N^{2}} \frac{\overline{\rho^{\prime}}}{\rho_{0}^{2}} \\
B T=-\left(\overline{u^{\prime 2}} \frac{\partial \bar{u}}{\partial x}+\overline{v^{\prime 2}} \frac{\partial \bar{v}}{\partial y}+\overline{u^{\prime} v^{\prime}} \frac{\partial \bar{u}}{\partial y}+\overline{u^{\prime} v^{\prime}} \frac{\partial \bar{v}}{\partial x}\right) \\
B C=-\frac{g^{2}}{\rho_{0}^{2} N^{2}}\left(\overline{\overline{u^{\prime} \rho^{\prime}}} \frac{\partial \bar{\rho}}{\partial x}+\overline{v^{\prime} \rho^{\prime}} \frac{\partial \bar{\rho}}{\partial y}\right) \\
K H=-\left(\overline{w^{\prime} u^{\prime}} \frac{\partial \bar{u}}{\partial z}+\overline{w^{\prime} v^{\prime}} \frac{\partial \bar{v}}{\partial z}\right)
\end{array}
$$

where $\rho$ is the density of sea water, $p$ the pressure, and $N^{2}$ the buoyancy frequency. In general the overline i.e. $\bar{u}$, can refer to the time mean but here will indicate the monthly climatology with primes i.e. $u^{\prime}$, denoting anomalies about the climatology. For the respective terms in Eqn. 3a, EKE is the transient or eddy kinetic energy and EPE the transient potential energy; BT and BC are the barotropic and baroclinic conversion terms. For BT positive, energy is drained from the mean horizontal shears to the eddy field whereas; for $\mathrm{BC}$ positive, energy is drained from the horizontal density gradients, equivalent to the mean available potential energy, to the eddy field. 
Contributions from the mean vertical shears and Reynolds stresses in the vertical plane are included in the Kelvin-Helmholtz (KH) instability. In order for conservation of energy transfers, release of mean kinetic energy (i.e. positive BT and $\mathrm{KH}$ ) must be accompanied by capture of potential energy (i.e. negative BC). The divergence (i.e., pressure work) term $-\nabla \cdot\left(\overline{\mathbf{v}^{\prime} p^{\prime} / \rho_{0}}\right)$ vanishes if integrated over a closed domain. We can define an additional exchange term, that, if positive, describes the drain of energy from EPE into EKE

$$
P K C=-\frac{g}{\rho_{0}}\left(\overline{\rho^{\prime} w^{\prime}}\right) .
$$

In the results to follow, due to the negligible changes to the vertical velocities $w$ in the experiments with stochastic forcing of the horizontal velocity tendencies, $\mathrm{KH}$ contributions will not be explicitly considered.

Following Oliver et al. (2015), we consider the time mean transient (eddy) kinetic energy EKE in Joules ( $\mathrm{J}$ ) within a volume $V$ in the modified form

$$
E K E=\frac{1}{2} \int_{V} \rho\left(\overline{u^{\prime 2}}+\overline{v^{\prime 2}}\right) d V
$$

Following O'Kane et al. (2013), the transfer rate of mean to transient potential energy representing baroclinic instabilities, in Joules per second $(\mathrm{J} / \mathrm{s})$, is now given by

$$
G P E=g \int_{V} \frac{\overline{u^{\prime} \rho^{\prime}} \frac{\partial \bar{\rho}}{\partial x}+\overline{v^{\prime} \rho^{\prime}} \frac{\partial \bar{\rho}}{\partial y}}{\frac{\partial \tilde{\rho}}{\partial z}} d V
$$

where $g$ is the acceleration due to gravity and $\tilde{\rho}$ is a reference state for the ocean approximated by the zonally and meridionally averaged density.

\section{1) TIMESERIES}

We first consider the global volume integrated, annual averages of the kinetic and potential energy and temperature (figure 3). The transient kinetic energy is determined by the anomalous velocities and, after a drammatic increase over the first decade, attains stable values after year 50 . The values of the response of the model to increasing amplitude of the perturbations is not quite linear with global values of approximately $7 \times 1 e^{17} \mathrm{~J}$ for the control and $17 \times 1 e^{17} \mathrm{~J}$ for the stochastic- 
10 simulation. For the potential energy, we see significant reductions of up to $8 \%$ and $30 \%$ for the simulations with the 50\% (stochastic-5) and 100\% (stochastic-10) amplitude perturbations. There is also a corresponding increase in global volume annual averages of ocean temperature such that at year 170, there are increases of approximately $4 \%$ and $12 \%$ for the stocahstic-5 and stochastic-10 simulations respectively, relative to the initial state. The increase in mean kinetic energy accompanied by decreasing potential energy is consistent with the earlier discussion of the energetics.

\section{2) KinETIC ENERGY SPECTRA}

The question arises as to the mechanism by which the energetics and temperature in the model respond to increasingly larger amplitude stochatic perturbations. As the stochastic forcing applied to the velocity tendencies has zero mean, we expect that energy is being redistributed across scales and not injected. The application of stochastic forcing to redistribute energy across disparate scales, with corresponding modifications to large scale flow structures, has been examined previously in the context of two-dimensional turbulence (Bouchet and Simonnet 2009; Loxley and Nadiga 2013; Nadiga and O'Kane 2017) but not to our knowledge for an ocean GCM. To better understand the redistribution of energy and the source of the increased transients in our low resolution ocean GCM, we next consider the kinetic energy spectra averaged across the global ocean. Specifically we consider total KE and it's component parts in terms of the triple decomposition (Hussain and Reynolds 1970; Kitsios et al. 2010)

$$
\zeta(x, t)=\langle\zeta(x)\rangle+\tilde{\zeta}(x, t)+\zeta^{\prime}(x, t)
$$

i.e. $\bar{\zeta}(x)=\langle\zeta(x)\rangle+\tilde{\zeta}(x, t)$ (climatology); $\tilde{\zeta}(x, t)$ (seasonal = climatology minus mean); $\zeta^{\prime}(x, t)$ (anomalies about the climatology); where the mean $\langle\zeta(x)\rangle=\frac{1}{T} \int_{0}^{T} \zeta(x, t) d t$ with $T$ the length of the timeseries.

In the top row of figure 4 we show the total $\mathrm{KE}$ and its constituent components i.e. mean, seasonal and anomalous KE. We show spectra calculated from velocities depth averaged to $1000 \mathrm{~m}$, noting that investigations at various depth levels in the upper ocean reveal a qualitatively similar picture. The total and mean KE spectra are closely matched for the control and all stochastically forced models indicating that the total energy remains largely conserved regardless of the strength of the 


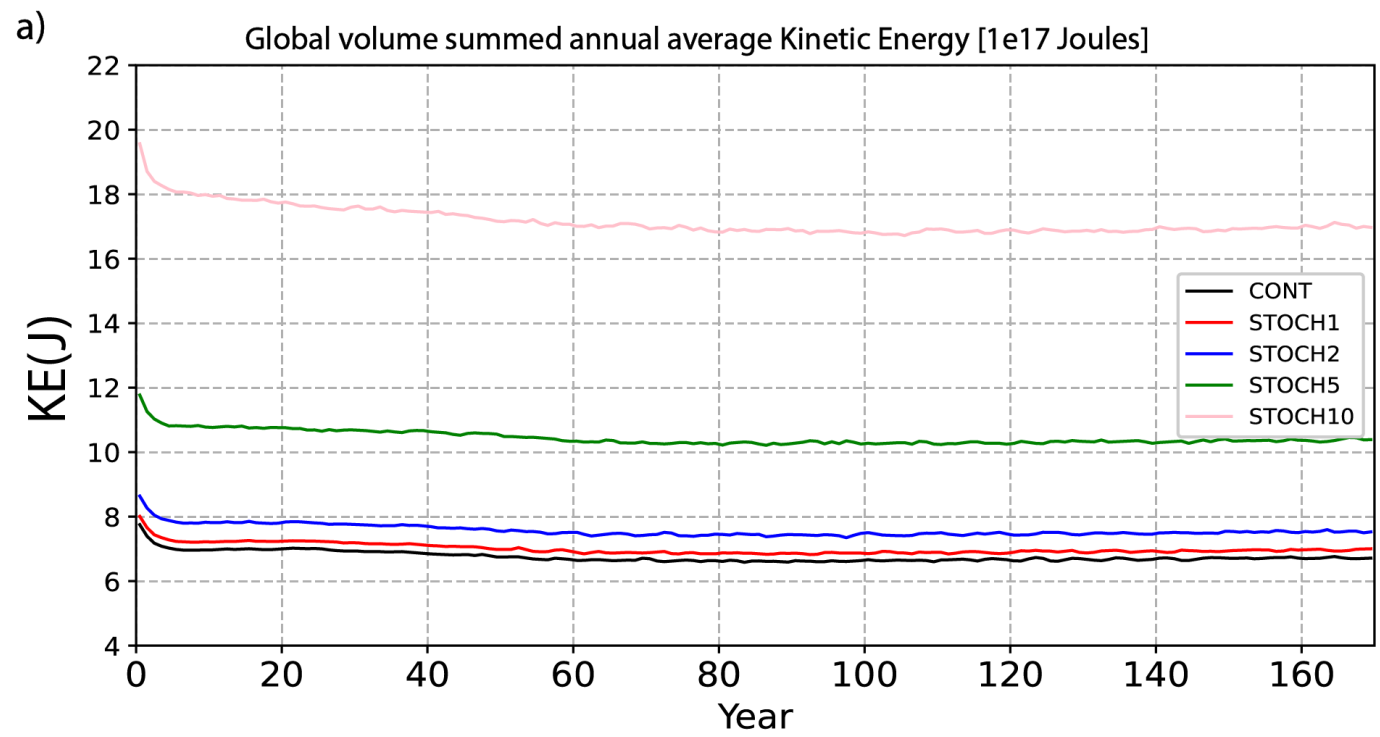

b) Global volume summed annual average Potential Energy [1 e6 (scaling)+27860 (offset) Joules]

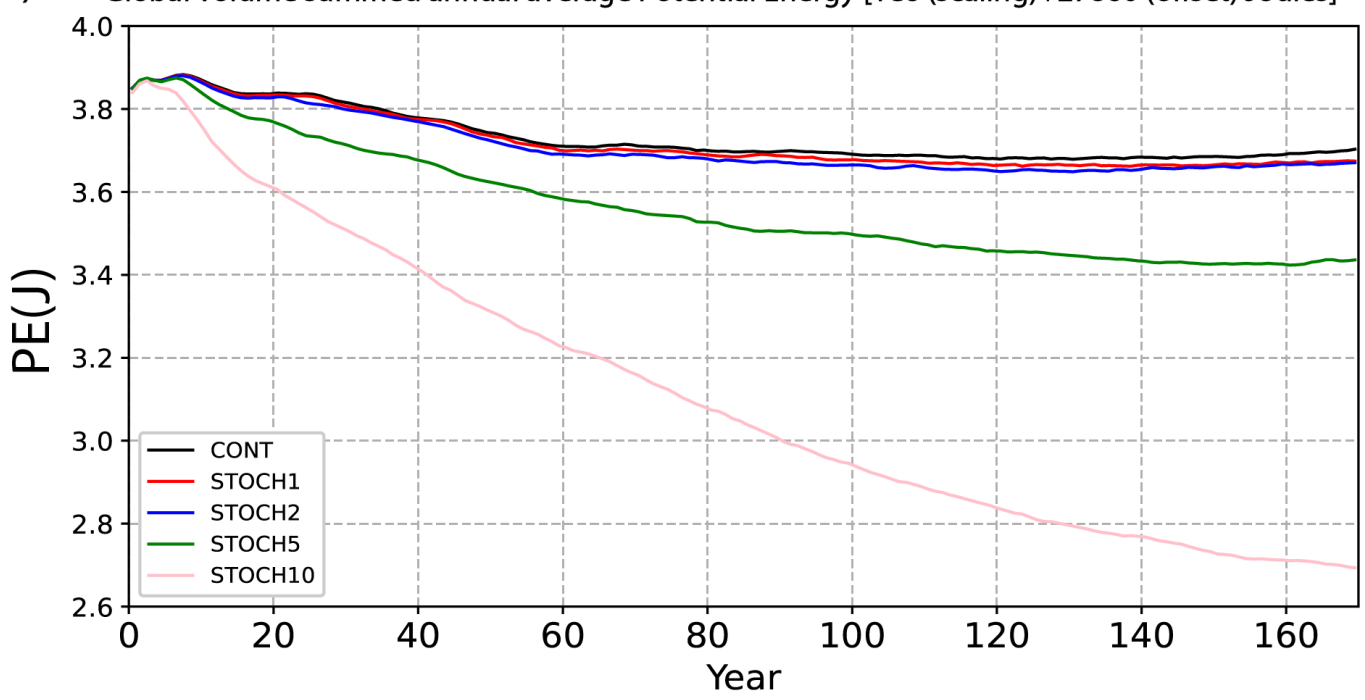

C) Global volume summed annual average Temperature [degrees Celcius]

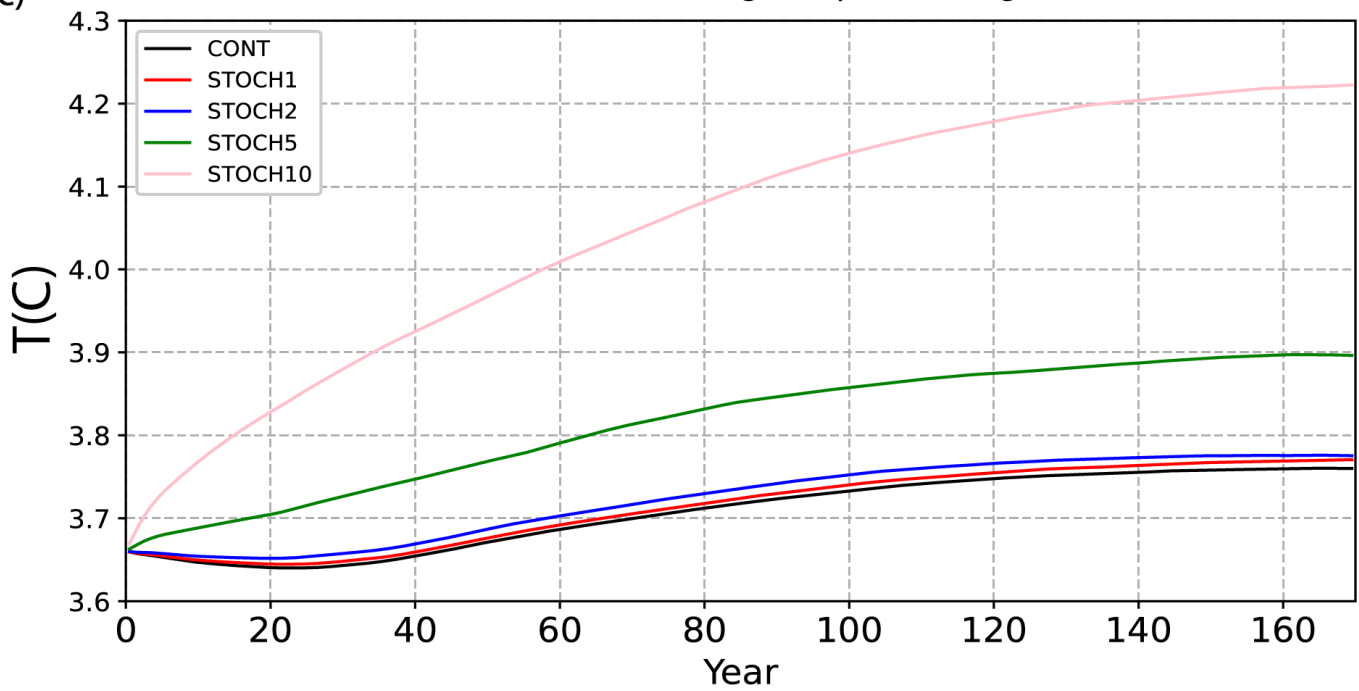

FIG. 3. Timeseries for globally summed KE, PE and temperature as annual averages. 
forcing. The interesting result, is that as the forcing amplitude is increased energy is transferred from the seasonal cycle (figure 4 top row third panel from the right) to generate transients (figure 4 top row last panel on the right) with transient KE being preferentially generated at the small scales i.e. total wavenumbers $n \in[10,100]$.

This observation is made even clearer when we consider the total, mean, seasonal and anomalous $\mathrm{KE}$ as a ratio between forced and control simulations (figure 4 second row). For the ratio of forced to control total, we see additional redistribution of KE from the large scales to the small scales relative to the control as the stochastic forcing amplitude increases. In contrast, for the mean and seasonal $\mathrm{KE}$ there is uniform transfer of $\mathrm{KE}$ to the transient anomalous $\mathrm{KE}$ across all scales but in particular to the smallest resolved scales. This transfer to the transients exhibits a cusp like functional form reminiscient of stochastic backscatter subgrid terms first dsescribed by Kraichnan (1976) for homogeneous turbulent flows. Where we consider KE transfers from the mean field to the transients at a given level (not shown) i.e. on a two-dimensional surface, the results are directly interpretable in terms of the results of O'Kane and Frederiksen (2008b) (see their figure 1 and figures $6 \mathrm{c} \& \mathrm{~d}$ ) and specifically momentum transfers due to the eddy-mean field (nonlinear noise and dissipation terms) and eddy-topographic force. The transfers from the large scale mean flow to generate small scale transients is even more clearly demonstrated when the mean, seasonal and anomalous KE is normalised by the total KE at each wavenumber (figure 4 row 3). For scales smaller than total wavenumber $n=50$, significant reductions in mean and seasonal KE occur relative to the total KE for the stochastic-5 (50\%) and stochastic-10 (100\%) simulations. For $n>10$, this energy is preferentially redistributed to the smaller scales however some of the mean and seasonal KE is uniformly transferred to the large scale structures i.e. $n \leq 10$. The extent to which stochastic forcing initiates energy transfers from the seasonal to the transients is revealed in the ratio of anomalous to seasonal KE (figure 4 bottom row).

\section{3) Transient KINETIC ENERGY AND BAROCLINIC INSTABILITY}

For a detailed examination of the energetics in physical space, we consider the transient kinetic energy (Eq. 5) and potential energy transfer (Eq. 6) at each grid point for the surface averaged over the last decade of the 170 year simulations. We first focus on the Southern Ocean and the ACC as a representative region of high eddy variability (figure 5). As expected, the $0.1^{\circ}$ reference calculation 

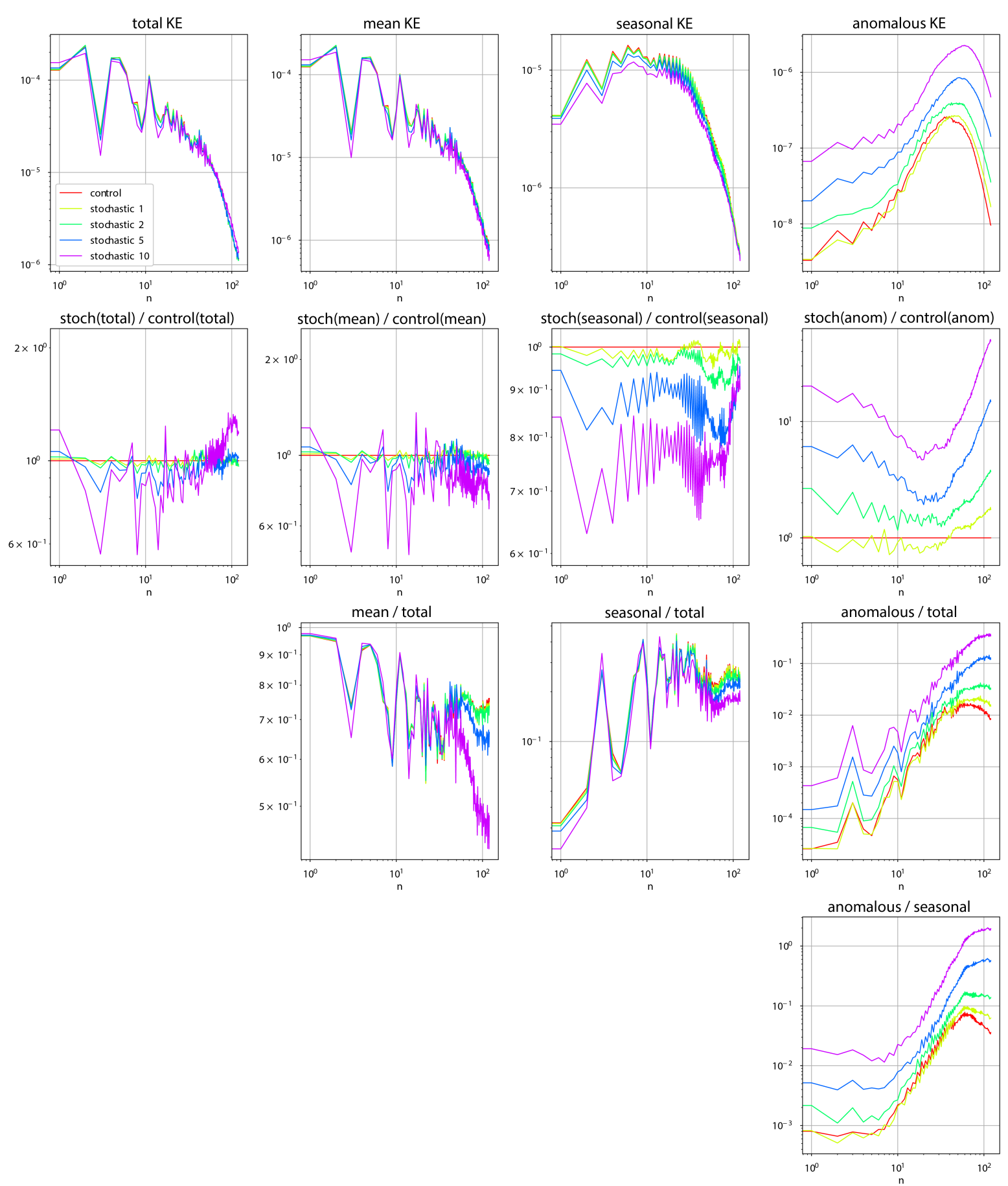

FIG. 4. Global KE spectra calculated from velocities depth averaged from $0-1000 \mathrm{~m}$ for the $1^{\circ}$ simulations. Shown in the top row are the total KE and the constituent terms of the triple-decomposition and in the second row as the ration of stochastically forced and control simulations. In row three we show the terms of the tripledecomposition normalised by the total $\mathrm{KE}$ and in the last row the ration of anomalous to seasonal $\mathrm{KE}$ for each of the $1^{\circ}$ simulations. 
show regions of high EKE throughout the ACC whereas for the $1^{\circ}$ control simulation EKE is largely absent. As the amplitude of the stochastic forcing increases so does EKE with initial responses colocated about large topographic features. The stochastic-10 (100\% amplitude) simulation displays comparable values and spatial distributions of EKE to the $0.1^{\circ}$ reference calculation.

Let us next consider baroclinic instability in the form of the transfer of mean to transient potential energy (GPE). We again focus on the Southern Ocean and the January average over the last simulated decade. In figure 5 , the $0.1^{\circ}$ reference calculation shows largely positive transfers in excess of $3 \mathrm{~J} / \mathrm{s}$ between $120^{\circ}-300^{\circ} \mathrm{E}$ with more uniformly distributed structures with values in excess of $\pm 3 \mathrm{~J} / \mathrm{s}$ elsewhere in the Atlantic and Indian ocean sectors. For the $1^{\circ}$ control simulation, similar meridionally oriented structures are present with typically much weaker values. With the addition of even very weak stochastic forcing (stochastic-1 10\%), structures with values comparable to the $0.1^{\circ}$ reference calculation appear. These structures become larger in extent and magnitude as the amplitude of the stochastic forcing increases and have previously been shown to be consistent with Rossby waves which can also be excited by the addition of noise directly to the surface forcing (O'Kane et al. 2013). O' Kane et al. (2014a) show that they, analagous to storm tracks in the midlatitude troposphere, are in fact higher order baroclinically unstable Rossby waves that propagate within wave guides defined by potential density gradients in the subtropical and higher latitude oceans.

In figure 6, we consider the zonal average GPE in the tropics, again for the January average over the last simulated decade. The $1^{\circ}$ control shows large-scale structures to $2000 \mathrm{~m}$ in depth North of $10^{\circ} \mathrm{N}$ with values exceeding $-4 \mathrm{~J} / \mathrm{s}$ with lower values extending only to $1000 \mathrm{~m}$ depth South of $10^{\circ}$ $\mathrm{S}$. The values betweem $\pm 10^{\circ}$ latitude are small-scale and weak. With the application of stochastic forcing, GPE values increase everywhere with larger scale structures appearing in the regions poleward of $10^{\circ}$ latitude and at greater depth in the South. For stochastic-5 (50\%) forcing, GPE values and structures in the equatorial regions closely match those of the $0.1^{\circ}$ reference calculation. As the latitude increases there are some structural differences between the $1^{\circ}$ simulations and the $0.1^{\circ}$ reference calculation, but with comparable values for the $50 \%$ and $100 \%$ forcing experiments. 
EKE
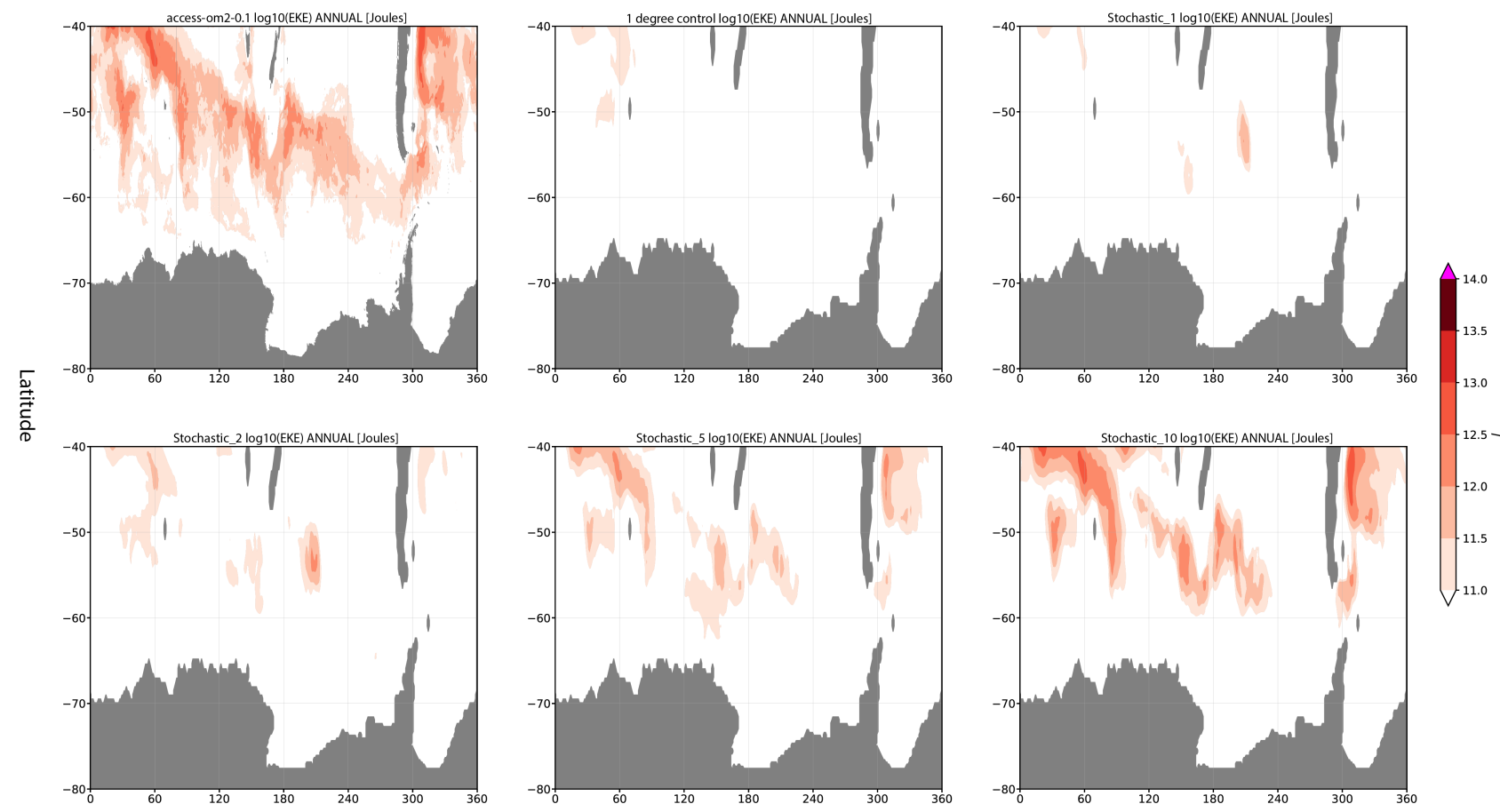

Longitude
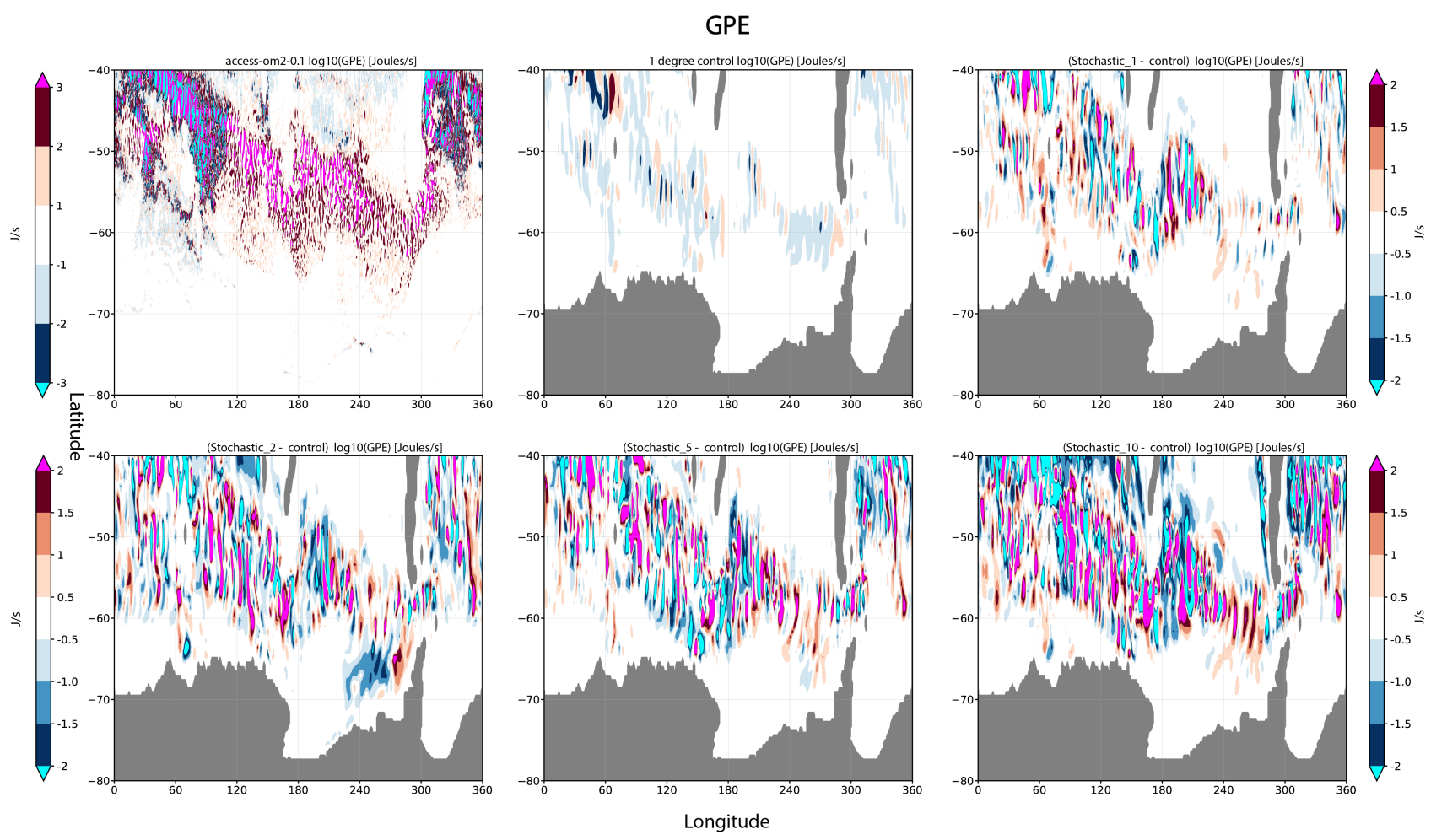

FIG. 5. Southern Ocean anomalous KE averaged (EKE) and transfer rate of mean to anomalous PE (GPE) in the Southern Ocean averaged for January over last decade of simulations. 

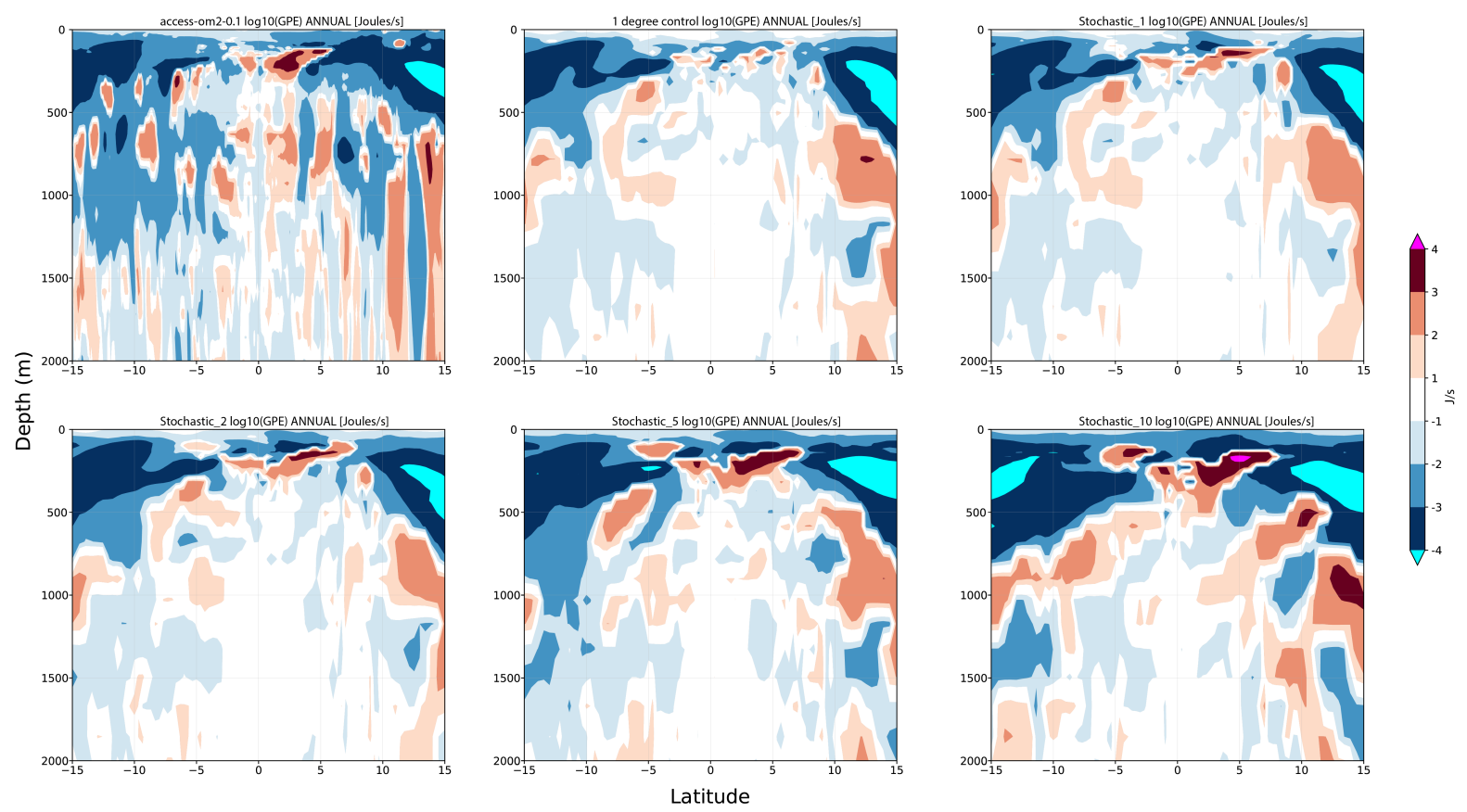

FIG. 6. Zonal average GPE in the tropics averaged for January over last decade of simulations.

\section{c. SST and MLD}

Given the observed transfer of energy from the mean and seasonal spectra to generate anomalous $\mathrm{KE}$, the question arises as to the spatial imprint on the dynamically active regions. We begin by first comparing January SST climatologies for the low resolution control simulation, the high resolution reference calculation, and differences between the forced simulations and control (figure 7). We first notice agreement between the high and low resolution simulations, in part expected due to the common surface boundary conditions but the general level of agreement is remarkable. With the application of stochastic forcing, we see the initial response in the mid-latitude boundary current regions of the North Pacific and Atlantic, once again notably in the regions associated with the Kuroshio extension and Gulf Steam separation.

For the stochastic-5 simulation, the Northern hemisphere responses are revealed as largely meridional displacements to the aforementioned boundary currents and in the Atlantic to the gyre circulation encompassing the North Atlantic drift and Canaries current. In the western Pacific, we see warming along the Alaska and California currents. In the Southern hemisphere, there is cooling in the East Australian Current, the South Equatorial, Mozambique and Agulhas Currents, the Falklands Current and regions in the ACC. 

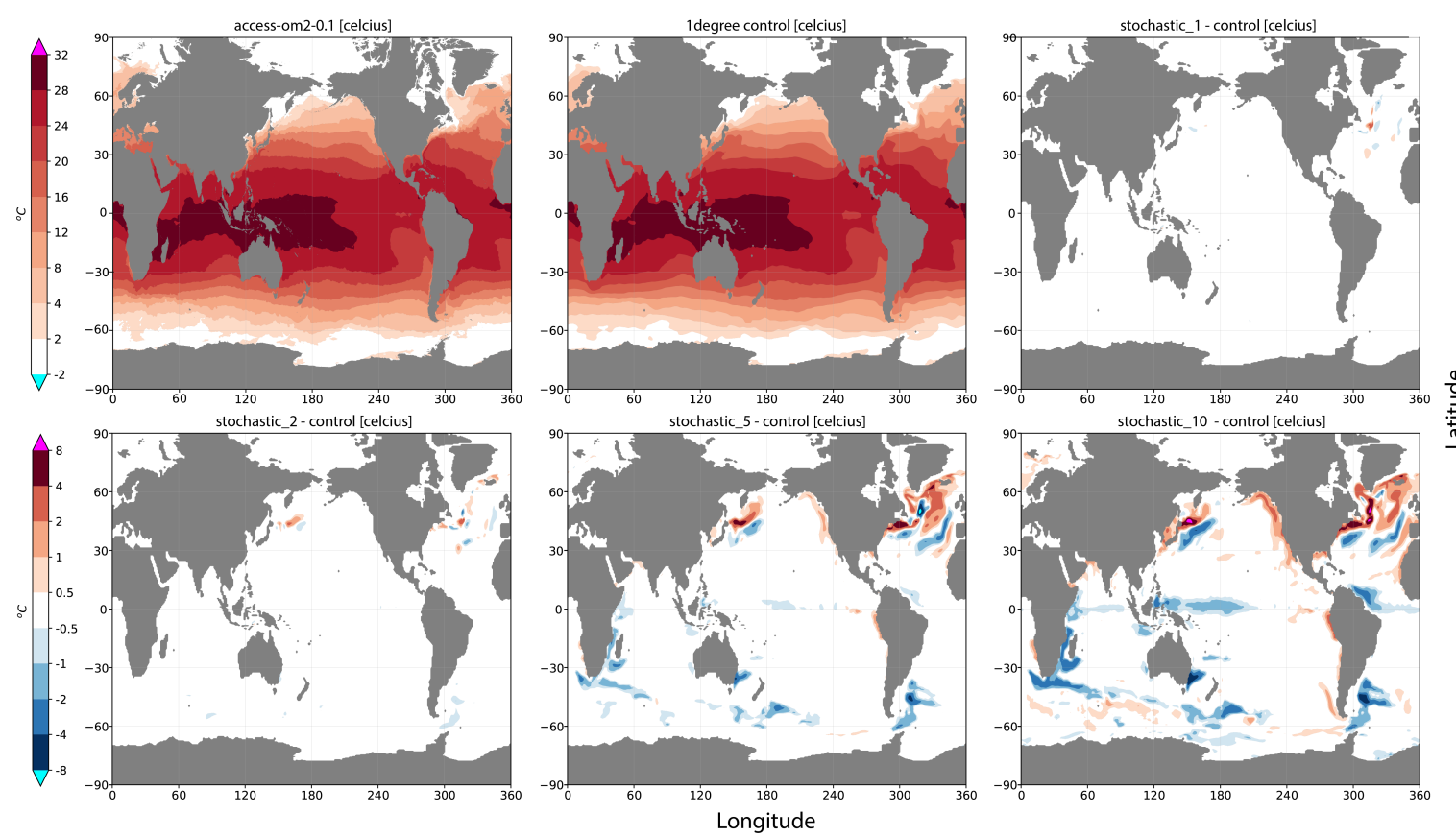

FIG. 7. High and low resolution reference calculations of SST and differences of stochastic forced w.r.t. control calculated for climatological January over the last decade of simulations.

At $100 \%$ amplitude stochastic forcing (stochastic-10) there is further amplification of the aforementioned responses but with additional cooling in the western equatorial Pacific. In contrast to substantial cooling (up to $4^{\circ} \mathrm{C}$ ) in the western equatorial Atlantic, warming is evident all along the Eastern coast of South America. The general patterns of warming and cooling in the Southern hemisphere are less representative of meridional displacement of currents and more indicative of changes to mixing processes. This is indeed shown to be the case in examination of the January climatological mixed layer depth (MLD) (figure 8). Of note is the substantial difference in MLD at the Kuroshio extension in the North Pacific at around $40^{\circ} \mathrm{S}$ between high resolution reference and low resolution control simulations. With increased forcing amplitudes, the meridional displacement of the currents in the Northern hemisphere are also shown to be accompanied by substantive changes in MLD. In the southern hemisphere, the cooling observed in the Southern Ocean is now revealed to occur primarily due to substantial increases in MLD of over 200m at locations where significant topographic features are located. This is indicative of increased momentum fluxes due to an enhancement of the eddy-topographic force. Considering the responses in July at the height of the austral winter (figure 8), substantive increases in MLD are observed throughout the ACC and, for maximum ampitude stochastic-10, at the Tasman Front extending from the Australian 
MLD
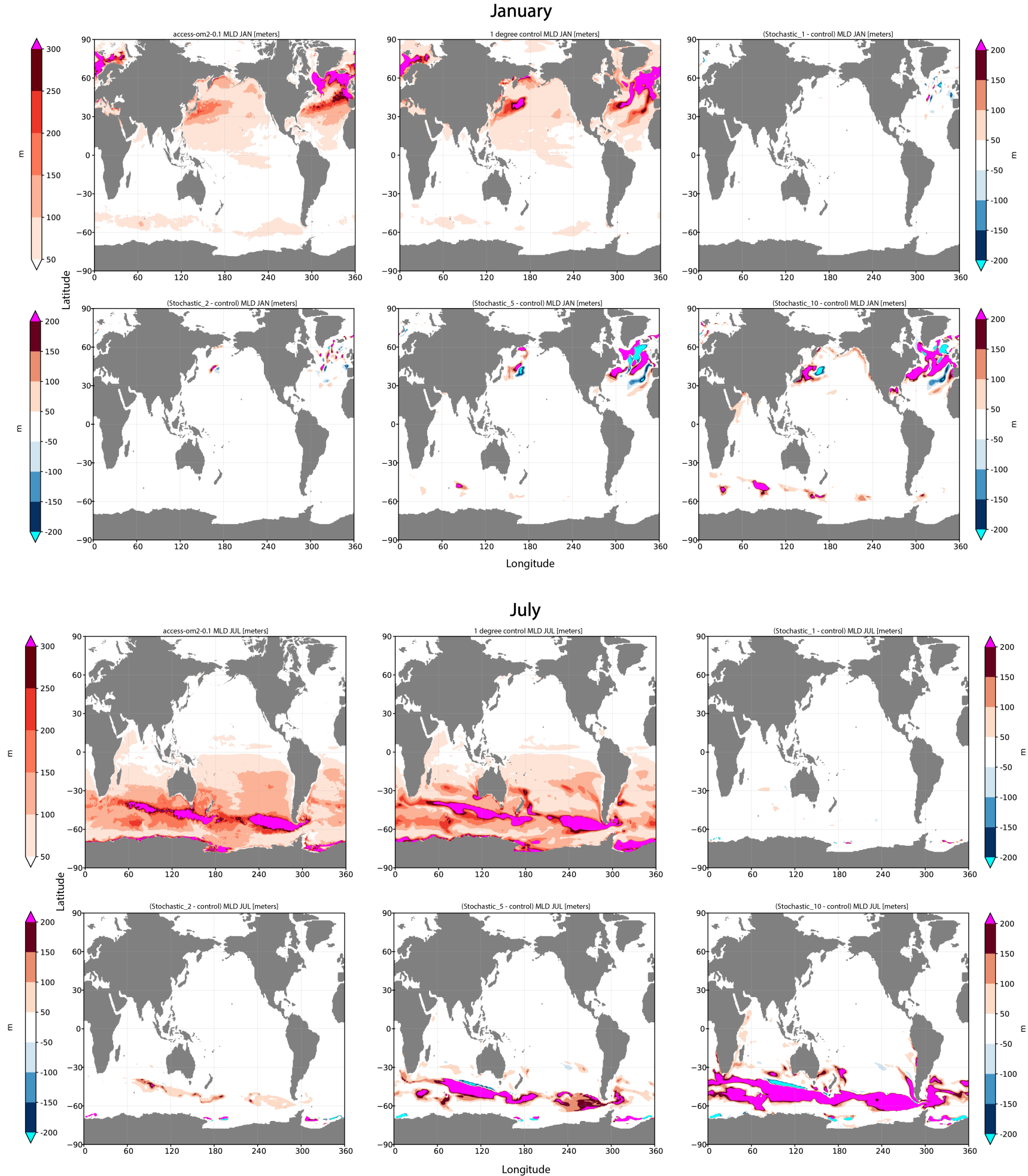

Fig. 8. High and low resolution reference calculations and differences of stochastic forced w.r.t. control calculated over last decade of simulations for January and July averaged mixed layer depths. 

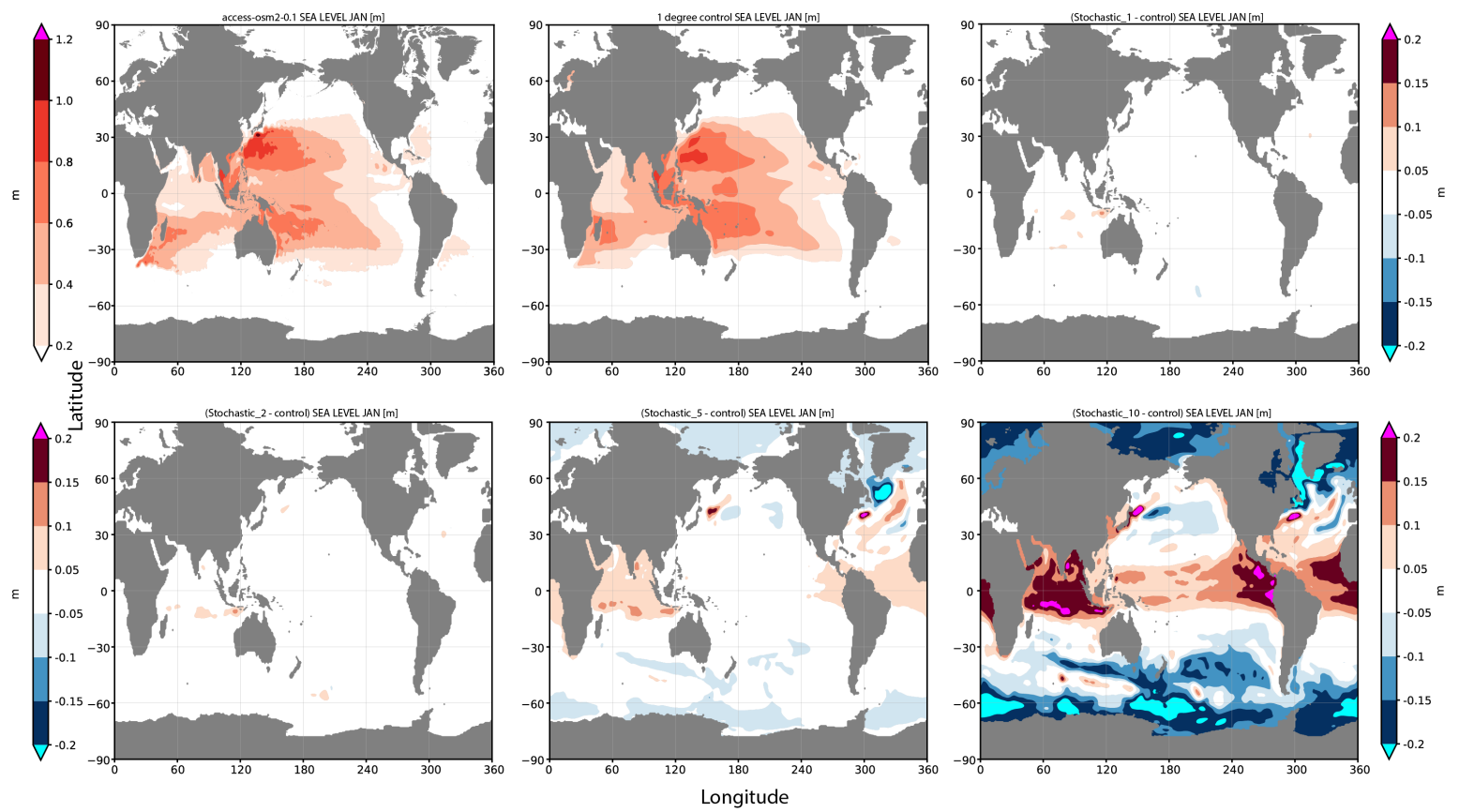

Fig. 9. High and low resolution reference calculations and differences of stochastic forced w.r.t. control calculated as a January climatological average over the last decade of simulations for sea level.

coast to the west of New Zealand and in the southern Atlantic in the region of the Brazil-Malvinas Confluence.

\section{d. Sea level, Temperature and $\mathrm{OHC}$}

Regions of substantive surface cooling can also be accompanied by subduction of large amounts of heat and local increases in sea level. This is exactly the case where the surface cooling previously observed in the equatorial oceans for large amplitude stochastic forcing (figure 7) is shown to be associated with increses in sea level of over $20 \mathrm{~cm}$ (figure 9 last panel) and anomalous temperature increases of more than 4 degrees at the thermocline (figure 10 last panel). Decreases in sea level occur in the mid-latitudes south of $30^{\circ} \mathrm{S}$ and at the high latitudes in the sea ice zones. These regions are however not associated with substantive surface (figure 7) or subsurface (figure 10) cooling rather, for the mid-latitude Southern Ocean, presumably occur due to increased mixing and an equatorward redistribution of heat to the tropics.

To better understand changes in sea level, we next consider ocean heat content (OHC) annually averaged globally and for the Atlantic, Pacific, Southern and Indian oceans through time, both integrated and by depth (figure 11 ), contrasting the $1^{\circ}$ control and stochastic-10 simulations. 

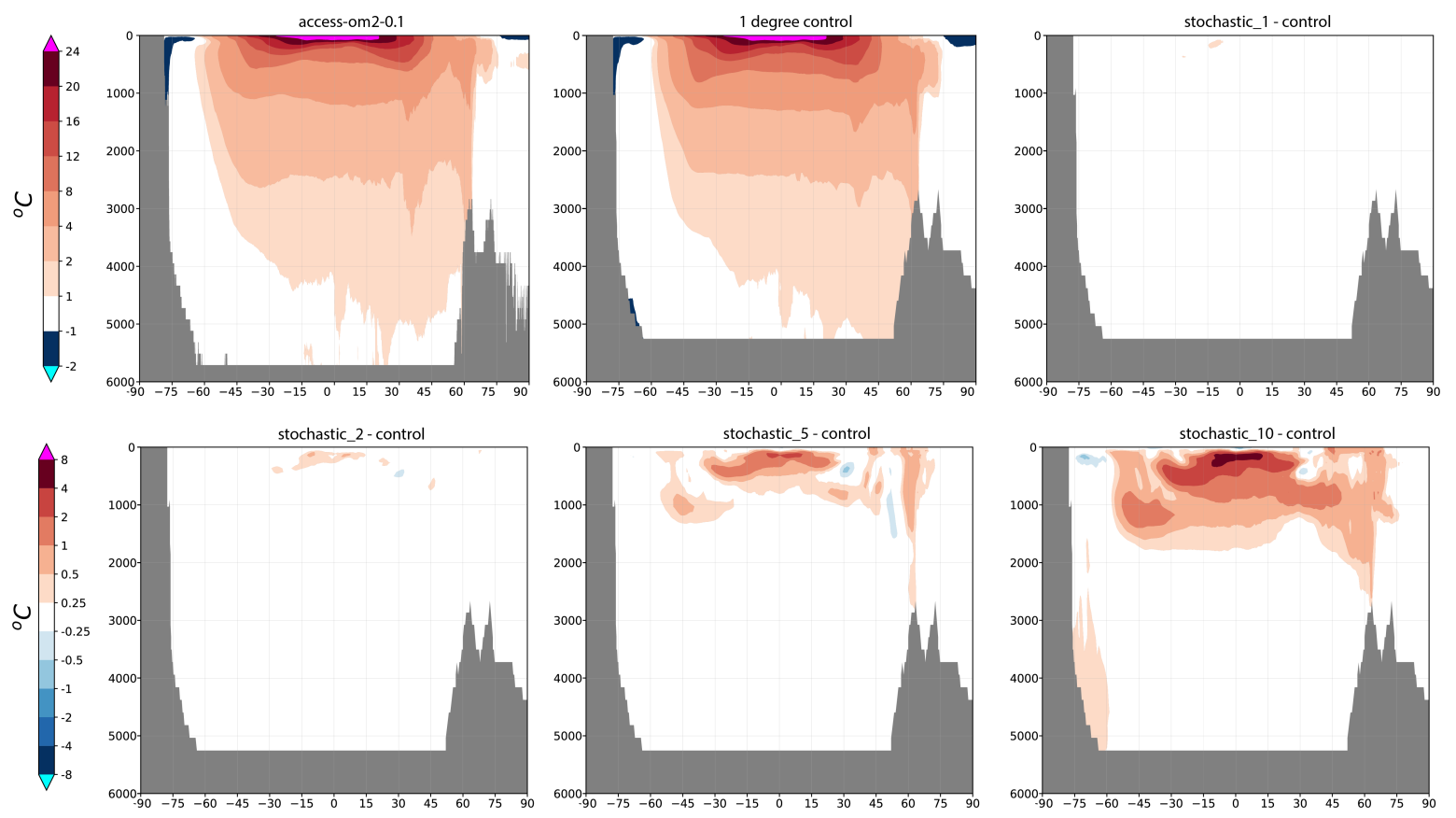

Fig. 10. High and low resolution reference calculations and differences of stochastic forced w.r.t. control calculated as a January climatological average over the last decade of simulations for zonal mean temperature.

The forced simulation requires around 150 years to reach a global steady state characterized by substantial increases in OHC at all depths but in particular above $2000 \mathrm{~m}$ and, with accompaning increases in the mean thermocline depth from $\approx 1500 \mathrm{~m}$ to $\approx 2500 \mathrm{~m}$. While the change in global $\mathrm{OHC}$ is very small, representing an increase of only $0.17 \%$ globally, it is significant reaching around $100 \times 10^{22} \mathrm{~J}$ after 40 years before equilibrating at $250 \times 10^{22} \mathrm{~J}$ after 150 years. The change to $\mathrm{OHC}$ caused by strong uncorrelated transient (eddy) noise is of the same magnitude but opposite sign to that observed by Williams et al. (2016) employing strong correlated noise perturbations applied to the temperature tendencies. Where Williams et al. (2016) also observed warming in the top $1000-2000 \mathrm{~m}$, they observed proportionally much larger cooling at depths between $3000-4000 \mathrm{~m}$, to the extent that there was a net cooling of the global ocean. In contrast, our results reveal warming at all depths with changes (units of $1 e 22 \mathrm{~J}$ ) in the Atlantic $\approx 55$, Southern $\approx 40$ and Indian $\approx 40$ oceans at year 170, and where nearly half of the total warming occurs in the Pacific $\approx 115$, mostly concentrated at the equator (see also figure 10). In contrast to Williams et al. (2016), our results show no evidence of cooling at depth. As noted earlier, all our simulations have reached steady state for global $\mathrm{OHC}$ after a transient period of $\approx 150$ years with no evidence of additional subduction of heat. 

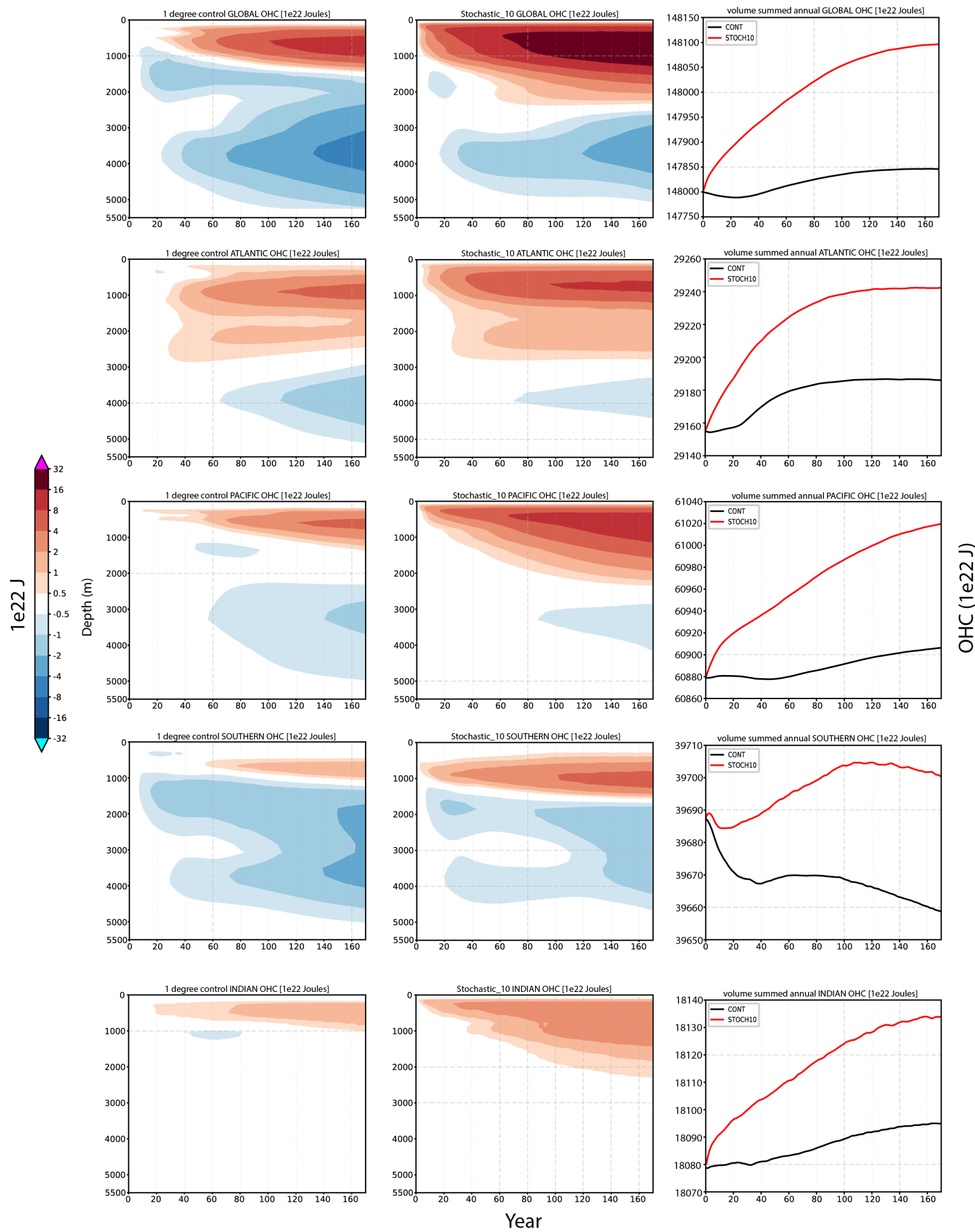

Fig. 11. Annually averaged ocean heat content (OHC) for the global, Atlantic, Pacific, southern and Indian oceans by depth and volume integrated. We show only the $1^{\circ}$ control and stochastic-10 simulations. 


\section{e. Transports}

Finally we are interested to see what impact the described changes in the climatological state have on ocean transports (figure 12). We consider transports for Drake Passage, the Atlantic Meridional Ocean Circulation at $26^{\circ} \mathrm{N}\left(\mathrm{AMOC} 26^{\circ} \mathrm{N}\right)$, Antarctic Bottom Water (AABW) and North Atlantic Deep water (NADW). The methodology used in the calculation of these transports has been described in detail in section 6d of O'Kane et al. (2021).

Drake Passage transport (figure 12) is a proxy for the strength of the ACC and is here calculated using monthly averaged 3D ocean horizontal mass transports from which the eastward component is integrated along a single line from the southern tip of South America to the northern tip of the Antarctic Peninsula and to the ocean bottom. Here the ACC strength for the $0.1^{\circ}$ high resolution reference simulation lies on average between $140-150 \mathrm{~Sv}$ and between $150-160 \mathrm{~Sv}$ in the $1^{\circ}$ control increasing to a maximum of between $155-165 \mathrm{~Sv}$ for stochastic-10 with a near linear response to increases in stochastic forcing amplitude. All simulations are within observational estimates of the observed Drake Passage transport values which range between $134 \pm 13 \mathrm{~Sv}$ (Whitworth and Peterson 1985) and 173 Sv (Donohue et al. 2016).

The $0.1^{\circ}$ high resolution reference transport for the AMOC cell is centered about the estimated observed transport of 17.2 Sv at $26^{\circ} \mathrm{N}$ (McCarthy et al. 2015) and within the observed range of seasonal variations between 10 and 25 Sv from the RAPID-WATCH (Smeed et al. 2015). However, the $1^{\circ}$ control reveals a much too weak AMOC $26^{\circ} \mathrm{N}$ transport with seasonal fluctuations of between 3.5-9.5 Sv. Stochastic forcing acts to increase the transport by up to $3.75 \mathrm{~Sv}$ to maximum steady state values of $12.5 \mathrm{~Sv}$ (stochastic-10). The $1^{\circ}$ control NADW intensity averages between 7.5 $-12 \mathrm{~Sv}$ whereas the high resolution reference ranges between 16-25 Sv. The stochastic-5 and -10 simulations both generate seasonally varying values of between $10-15 \mathrm{~Sv}$ comparable to the observed values ranging about $\approx 15$ Sv (Lumpkin et al. 2008; Ganachaud 2003).

In the Southern Ocean, observed values of the AABW cell transports range from 5.6 $\pm 3.0 \mathrm{~Sv}$ reported by Lumpkin and Speer (2007) to values of 9.77 $\pm 3.7 \mathrm{~Sv}$ reported in the Weddell Sea (Sloyan and Rintoul 2001; Garabato et al. 2002; Talley 2013). In figure 12, the $0.1^{\circ}$ high resolution reference AABW transports lie within the range observed by Lumpkin and Speer (2007), whereas the $1^{\circ}$ control simulation values are much closer to those reported by Talley (2013) for example. 

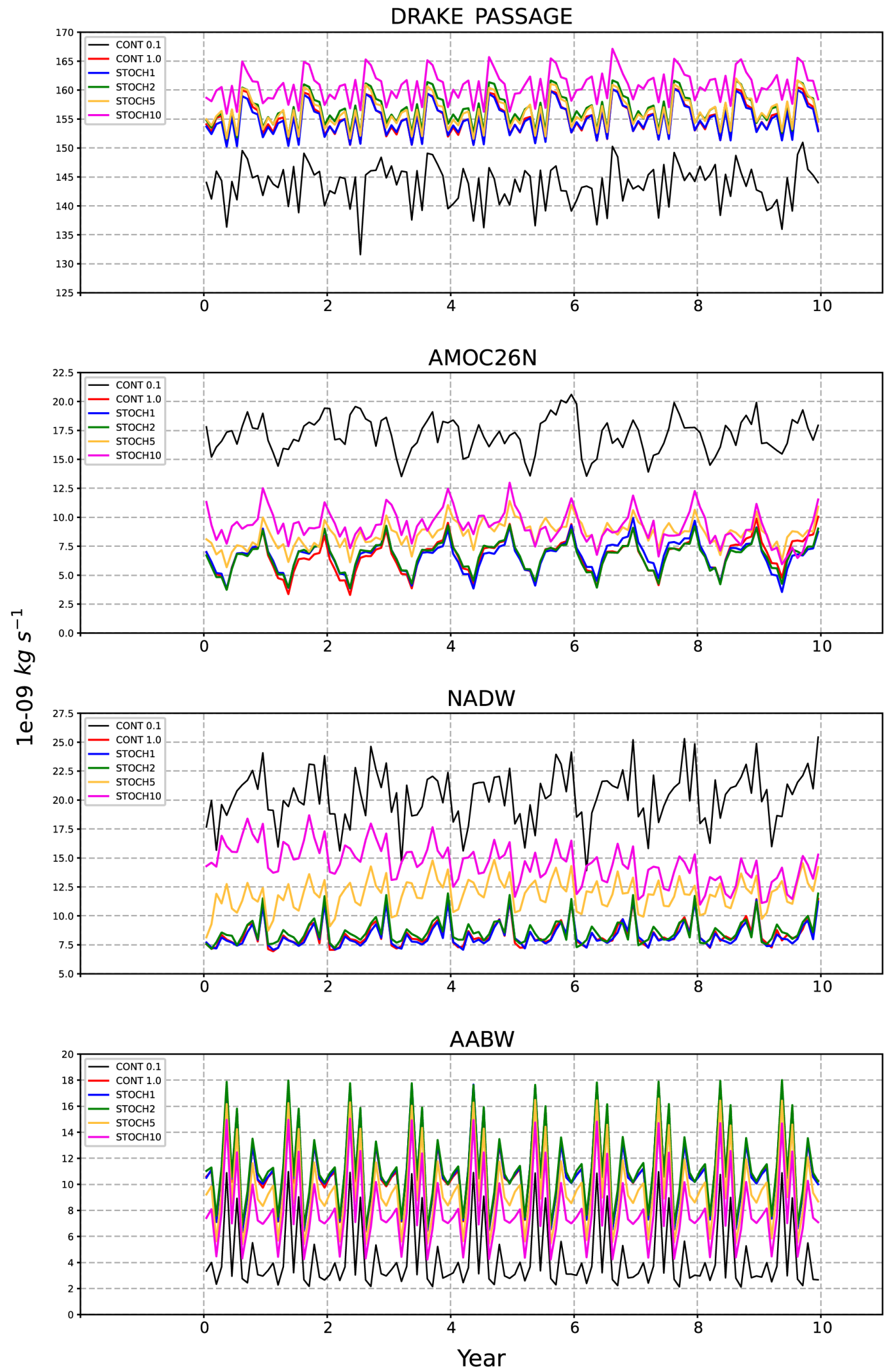

FIG. 12. Comparison of transports for Drake Passage, AMOC26 N, NADW and AABW. 
Here the impact of increased noise is to reduce the average transport to be closer to the high resolution reference values.

Thus, we see that the addition of noise is not to always increase transports, but, with the exception of Drake Passage, to most often to make the dynamics of the low resolution non-eddying model more consistent with that of the high resolution eddying model, whether that be to increase or decrease volume transports. Apart from AABW, the $0.1^{\circ}$ reference calculation transports display less regular seasonal variability, despite having the same repeat forcing applied as the $1^{\circ}$ degree simulations. We ascribe this to the presence of randomly generated eddies with deep vertical extent in the high resolution reference model but have not undertaken a rigorous examination of this point.

\section{Summary and Discussion}

Overall, we find implementation of a simple parameterization of ocean transients (eddies), via stochastic perturbations to the horizontal momentum fluxes, leads to improvements in the simulated climatological steady intrinsic ocean state. The statistics of the transients were calculated from the velocities of a high-resolution, eddy-resolving ocean model ACCESS-OM2-0.1. After thresholding, a three-dimensional mask was generated enabling the injection of stochastic noise i.e., zero-mean random noise uniformly distributed between $[-1,1]$, representative of subgrid transients, into a low-resolution, $1^{\circ}$ non-eddy-resolving variant of the same ocean - sea ice model configuration. Four variants of the stochastically forced $1^{\circ}$ ACCESS-OM 2 model were considered, with varying amplitudes of the noise relative to the high resolution reference calculation applied. All low resolution model configurations were run to steady state before calculation of the statistics of their respective climatological states.

Spectra from a triple-decomposition revealed that, despite having zero-mean, random noise forcing was able initiate a redistribution of kinetic energy largely from seasonal variations to generate large amplitude small scale anomalous transient kinetic energies. A major improvement was observed in the energetics of the Southern Ocean where the transient kinetic energy of the Antarctic Circumpolar Current, largely absent in the $1^{\circ}$ control simulation, was able to be approximated to large degree with amplitudes matching those simulated in the $0.1^{\circ}$ reference calculation for sufficiently strong stochastic forcing. Similar stochastic amplification was observed in the transfer rate of mean to transient potential energy at all latitudes. 
Surface temperature responses were largely consistent with increases in mixed layer depths and meridional displacement of northern hemisphere boundary currents. Decreases in sea level at the higher latitudes, compensated by increased sea levels at the equator, were found to be largely in response to injection of heat into the equatorial Pacific at the thermocline and into the mixed layer. While consistent warming was observed at all depths, by far the majority of the OHC warming occurred in the equatorial Pacific upper ocean. Improvements in the transports include important overturning circulations such as increases in strength of the AMOC $26^{\circ} \mathrm{N}$ and NAWDW, and weakening of AABW. Only Drake Passage transport moved further from the high resolution reference calculation but remained withing the range of observational estimates. While the maximum amplitudes of $\mathrm{OHC}$ differences between control and stochastic forcing experiments were comparable to those observed by Williams et al. (2016) using pertubed temperature tendencies, stochastic perturbations to the momentum fluxes produced global $\mathrm{OHC}$ warming whereas perturbed temperature tendencies produced cooling of the total OHC. Both responses can be at least as large in amplitude as the observed anthropogenic global warming signal.

Consistent with Williams et al. (2016) and the atmospheric study of Berner et al. (2012), we find the addition of stochastic forcing can result in improvements comparable to significant increases in horizontal resolution. We note that application of perturbations to the temperature tendencies alone will be inconsistent with modifying the potential energy and inappropriate in regions of density compensation, hence our motivation for modifying the momentum fluxes via the velocity tendencies. Overall we advocate for oceanic stochastic parameterizations as a simple and effective means to improve climate model simulations.

Acknowledgments. The authors acknowledge support from National Computational Infrastructure (NCI) Australia. We also acknowledge the combined efforts of the Consortium for Ocean - Sea Ice Modelling in Australia (COSIMA) in developing the model configurations used in this study.

Data availability statement. All model configurations are available from the COSIMA github repository https://github.com/COSIMA/access-om2. The data and analysis codes are available on request. 


\section{References}

Andrejczuk, M., F. C. Cooper, S. Juricke, T. N. Palmer, A. Weisheimer, and L. Zanna, 2016: Oceanic stochastic perturbations in a seasonal forecast system. Mon. Wea. Rev., 144, 18671875, doi:10.1175/MWR-D-15-0245.1.

Beena, B. S., and J.-S. von Storch, 2009: Effects of fluctuating daily surface fluxes on the time-mean oceanic circulation. Clim. Dyn., 33, 1-18, doi:10.1007/s00382-009-0575-y.

Berner, J., and coauthors, 2017: Stochastic parameterization: Toward a new view of weather and climate models. BAMS, 25, 565-587, doi:10.1175/BAMS-D-15-00268.1.

Berner, J., T. Jung, and T. N. Palmer, 2012: Systematic model error: The impact of increased horizontal resolution versus improved stochastic and deterministic parameterizations. J. Climate, 25, 4946-4962, doi:10.1175/JCLI-D-11-00297.1.

Bouchet, F., and E. Simonnet, 2009: Random changes of flow topology in two-dimensional and geophysical turbulence. Physical Review Letters, 109 (2), 094504.

Chapman, C. C., B. M. Sloyan, T. J. O’Kane, and M. A. Chamberlain, 2020: Interannual subtropical indian ocean variability due to long baroclinic planetary waves. J. Climate, 33, 6765-6791, doi: 10.1175/JCLI-D-19-0469.1.

Donohue, K. A., K. L. Tracey, D. R. Watts, M. P. Chidichimo, and T. K. Chereskin, 2016: Mean Antarctic Circumpolar Current transport measured in Drake Passage. Geophysical Research Letters, 43, 11 760-11 767, doi:10.1002/2016GL070319.

Epstein, E. S., 1969: Stochastic dynamic prediction. Tellus, 21, 739-759.

Epstein, E. S., and E. J. Pitcher, 1972: Stochastic analysis of meteorological fields. J. Atmos. Sci., 29, 244-257.

Fleming, R. J., 1971a: On stochastic dynamic prediction. I: The energetics of uncertainty and the question of closure. Mon. Wea. Rev., 99, 851-872.

Fleming, R. J., 1971b: On stochastic dynamic prediction. II: Predictability and utility. Mon. Wea. Rev., 99, 927-938. 
Frankignoul, C., and K. Hasslemann, 1977: Stochastic climate models, Part II. Application to sea-surface temperature anomalies and thermocline variability. Tellus, 29, 289-305, doi: 10.3402/tellusa.v29i4.11362.

Franzke, C. L. E., T. J. O’Kane, J. Berner, P. D. Williams, and V. Lucarini, 2015: Stochastic climate theory and modeling. Wiley Interdiscip. Rev.: Climate Change, 6, 63-78, doi:10.1002/wcc.318.

Frederiksen, J. S., 1999: Subgrid-scale parameterizations of the eddy-topographic force, eddy viscosity and stochastic backscatter for flow over topography. J. Atmos. Sci., 56, 1481-1494.

Ganachaud, A., 2003: Large-scale mass transports, water mass formation, and diffusivities estimated from world ocean circulation experiment (WOCE) hydrographic data. J. Geophys. Res., 108, doi:10.1029/2002JC001565.

Garabato, A. C. N., E. L. McDonagh, D. P. Stevens, K. J. Heywood, and R. J. Sanders, 2002: On the export of Antarctic Bottom Water from the Weddell Sea. Deep-Sea Research II, 49, $4715-4742$.

Hasslemann, K., 1976: Stochastic climate models. Part i Theory. Tellus, 28A, 473-485, doi: 10.1111/j.2153-3490.1976.tb00696.x.

Horenko, I., 2010: On the identification of nonstationary factor models and their application to atmospheric data analysis. J. Atmos. Sci., 67, 1559-1574, doi:10.1175/2010JAS3271.1.

Hussain, A. K. M. F., and W. C. Reynolds, 1970: The mechanisms of an organised wave in a turbulent shear flow. J. Fluid Mech., 41, 241-261.

Kalnay, E., 2003: Atmospheric modeling, data assimilation and predictability. Cambridge University Press.

Kiss, A. E., and Coauthors, 2020: ACCESS-OM2 v1.0: a global ocean-sea ice model at three resolutions. Geosci. Model Dev., 13, 401-442, doi:10.5194/gmd-13-401-2020.

Kitsios, V., L. Cordier, J. P. Bonnet, A. Ooi, and J. Soria, 2010: Development of a nonlinear eddy-viscosity closure for the triple-decomposition stability analysis of a turbulent channel. $J$. Fluid Mech., 664, 74-107, doi:10.1017/S0022112010003617. 
Kitsios, V., J. S. Frederiksen, and M. J. Zidikheri, 2016: Theoretical comparison of subgrid turbulence in atmospheric and oceanic quasi-geostrophic models. Nonlinear Processes in Geophysics, 23, 95-105.

Kraichnan, R., 1976: Eddy viscosity in two and three dimensions. J. Atmos. Sci., 33, 1521-1536.

Lou, J., T. J. O'Kane, and N. J. Holbrook, 2021: A linear inverse model of tropical and south pacific climate variability: Optimal structure and stochastic forcing. J. Climate, 34, 143-155, doi:10.1175/JCLI-D-19-0964.1.

Loxley, P. N., and B. T. Nadiga, 2013: Bistability and hysteresis of maximum-entropy states in decaying two-dimensional turbulence. Physics of Fluids, 25 (1), 015113.

Lumpkin, R., and K. Speer, 2007: Global ocean meridional overturning. J. Phys. Oceanogr., 37, $2550-2562$.

Lumpkin, R., K. Speer, and K. Koltermann, 2008: Transport across $48^{\circ} \mathrm{N}$ in the Atlantic Ocean. $J$. Phys. Oceanogr., 38, 733-752.

Madec, G., and the NEMO team, 2016: NEMO ocean engine. Note du Pôle de modélisation, Institut Pierre-Simon Laplace (IPSL) No 27, ISSN No 1288-1619, URL https://www.nemo-ocean.eu/ doc/.

McCarthy, G., and Coauthors, 2015: Measuring the Atlantic meridional overturning circulation at $26^{\circ}$ N. Progress in Oceanography, 130, 91-111, doi:10.1016/j.pocean.2014.10.006.

Metzner, P., L. Putzig, and I. Horenko, 2012: Analysis of persistent nonstationary time series and applications. Comm. App. Math. Comp. Sci., 7, 175-229, doi:10.2140/camcos.2012.7.175.

Miller, R. N., M. Ghil, and F. Gauthiez, 1985: Data assimilation in strongly nonlinear systems. $J$. Atmos. Sci., 51, 1037-1056.

Nadiga, B. T., and T. J. O'Kane, 2017: Nonlinear and stochastic climate dynamics. Low-frequency regime transitions and predictability of regimes in a barotropic model, C. L. E. Franzke, and T. J. O’Kane, Eds., Cambridge University Press, chap. 5, 136-158, doi:10.1017/9781316339251.

Oey, L. Y., 2007: Loop current and deep eddies. J. Phys. Oceanogr., 38, 1426-1449, doi:10.1175/ 2007JPO3818.1. 
O'Kane, T. J., and J. S. Frederiksen, 2008a: A comparison of statistical dynamical and ensemble prediction methods during blocking. J. Atmos. Sci., 65, 426-447, doi:10.1175/2007JAS2300.1.

O'Kane, T. J., and J. S. Frederiksen, 2008b: Statistical dynamical subgrid-scale parameterizations for geophysical flows. Physica Scripta, T132, 014033.

O’Kane, T. J., R. J. Matear, M. A. Chamberlain, and P. R. Oke, 2014b: ENSO regimes and the late 1970's climate shift: The role of synoptic weather and South Pacific ocean spiciness. J. Comp. Phys., 271, 19-38, doi:10.1016/j.jcp.2013.10.058.

O’Kane, T. J., R. J. Matear, J. S. Risbey, B. M. Sloyan, and I. Horenko, 2013: Decadal variability in an OGCM Southern Ocean: Intrinsic modes, forced modes and metastable states. Ocean Modelling, 69, 1-21, doi:10.1016/j.ocemod.2013.04.009.

Oliver, E. C. J., T. J. O’Kane, and N. J. Holbrook, 2015: Projected changes to tasman sea eddies in a future climate. J. Geophys. Res. Oceans, 120, 1-16, doi:10.1002/2015JC010993.

Orlanski, I., and M. D. Cox, 1973: Baroclinic instability in ocean currents. Geophys. Fluid Dyn., 4, 297-332.

O’Kane, T. J., R. J. Matear, M. A. Chamberlain, E. C. J. Oliver, and N. J. Holbrook, 2014a: Storm tracks in the southern hemisphere subtropical oceans. J. Geophys. Res. Oceans, 119, 6078-6100, doi:10.1002/2014JC009990.

O’Kane, T. J., P. R. Oke, and P. A. Sandery, 2011: Predicting the East Australian Current. Ocean Modelling, 38, 251-266, doi:10.1016/j.ocemod.2011.04.003.

O'Kane, T. J., and Coauthors, 2021: Cafe60v1: A 60-year large ensemble climate reanalysis. Part II: Evaluation. J. Climate, 34, 1571-1594.

Penland, C., 1989: Random forcing and forecasting using principal oscillation pattern analysis. Mon. Wea. Rev., 117, 2165-2185, doi:10.1175/1520-0493(1989)117,2165:RFAFUP.2.0.CO;2.

Penland, C., and P. D. Sardeshmukh, 1995: The optimal growth of tropical sea surface temperature anomalies. J. Climate, 8, 1999-2024, doi:10.1175/1520-0442(1995)008,1999:TOGOTS.2.0. $\mathrm{CO} ; 2$. 
Pitcher, E. J., 1977: Application of stochastic dynamic prediction to real data. J. Atmos. Sci., 34, $3-21$.

Quinn, C., D. Harries, and T. J. O'Kane, 2021: Dynamical analysis of a reduced model for the north atlantic oscillation. J. Atmos. Sci., 78, 1671, doi:10.1175/JAS-D-20-0282.1.

Sloyan, B. M., and S. R. Rintoul, 2001: The Southern Ocean limb of the global deep overturning circulation. J. Phys. Oceanogr., 31, 143-173.

Smeed, D., G. McCarthy, D. Rayner, B. I. Moat, W. E. Johns, M. O. Baringer, and C. S. Meinen, 2015: Atlantic meridional overturning circulation observed by the RAPID-MOCHA-WBTS (RAPID-Meridional Overturning Circulation and heatflux array-western boundary time series) array at 26N from 2004 to 2014. British Oceanographic Data Centre - Natural Environment Research Council, doi:10/6qb.

Stewart, K., and Coauthors, 2020: JRA55-do-based repeat year forcing datasets for driving ocean-sea-ice models. Ocean Modelling, 147, 101 557, doi:10.1016/j.ocemod.2019.101557.

Talley, L. D., 2013: Closure of the global overturning circulation through the Indian, Pacific, and Southern Oceans: Schematics and transports. Oceanography, 26, 80-97, doi:10.5670/oceanog. 2013.07.

Whitworth, T., and R. Peterson, 1985: Volume transport of the Antarctic Circumpolar Current from bottom pressure measurements. J. Phys. Oceanogr., 15, 810-816.

Williams, P. D., 2012: Climatic impacts of stochastic fluctuations in air-sea fluxes. Geophys. Res. Lett., 39, L10 705, doi:10.1029/2012GL051813.

Williams, P. D., N. J. Howe, J. M. Gregory, R. S. Smith, and M. J. Joshi, 2016: Improved climate simulations through a stochastic parameterization of ocean eddies. J. Climate, 29, 8763-8781, doi:10.1175/JCLI-D-15-0746.1.

Zavala-Garay, J., A. M. Moore, C. L. Perez, and R. Kleeman, 2003: The response of a coupled model of ENSO to observed estimates of stochastic forcing. J. Climate, 16, 2827-2842.

Zhou, Y., 2021: Turbulence theories and statistical closure approaches. Physics Reports, 935, 1-117, doi:10.1016/j.physrep.2021.07.001. 
Zidihkeri, M., and J. S. Frederiksen, 2008: Stochastic subgrid-scale modelling for non-equilibrium ${ }_{633}$ geophysical flows. Philos. Trans. Roy. Soc. London, 36A, 145-160, doi:10.1098/rsta.2009.0192. 